\title{
Left-inverses of fractional Laplacian and sparse stochastic processes
}

\author{
Qiyu Sun · Michael Unser
}

Received: 21 July 2010 / Accepted: 4 September 2010 /

Published online: 27 September 2011

(C) The Author(s) 2011. This article is published with open access at Springerlink.com

\begin{abstract}
The fractional Laplacian $(-\triangle)^{\gamma / 2}$ commutes with the primary coordination transformations in the Euclidean space $\mathbb{R}^{d}$ : dilation, translation and rotation, and has tight link to splines, fractals and stable Levy processes. For $0<\gamma<d$, its inverse is the classical Riesz potential $I_{\gamma}$ which is dilationinvariant and translation-invariant. In this work, we investigate the functional properties (continuity, decay and invertibility) of an extended class of differential operators that share those invariance properties. In particular, we extend the definition of the classical Riesz potential $I_{\gamma}$ to any noninteger number $\gamma$ larger than $d$ and show that it is the unique left-inverse of the fractional Laplacian $(-\triangle)^{\gamma / 2}$ which is dilation-invariant and translationinvariant. We observe that, for any $1 \leq p \leq \infty$ and $\gamma \geq d(1-1 / p)$, there exists a Schwartz function $f$ such that $I_{\gamma} f$ is not $p$-integrable. We then introduce the new unique left-inverse $I_{\gamma, p}$ of the fractional Laplacian $(-\triangle)^{\gamma / 2}$ with the property that $I_{\gamma, p}$ is dilation-invariant (but not translation-invariant) and that $I_{\gamma, p} f$ is $p$-integrable for any Schwartz function $f$. We finally apply that linear operator $I_{\gamma, p}$ with $p=1$ to solve the stochastic partial differential equation $(-\Delta)^{\gamma / 2} \Phi=w$ with white Poisson noise as its driving term $w$.
\end{abstract}

Communicated by Charles Micchelli.

Research of first author was supported by the National Science Foundation under grant DMS-1109063. Research of second author was supported by the Swiss National Science Foundation under grant 200020-121763.

Q. Sun $(\bowtie)$

Department of Mathematics, University of Central Florida, Orlando, FL 32816, USA

e-mail: qiyu.sun@ucf.edu

\section{Unser}

Biomedical Imaging Group, École Polytechnique Fédérale de Lausanne,

Lausanne 1015, Switzerland

e-mail: michael.unser@epfl.ch 
Keywords Fractional Laplacian • Riesz potential - Impulsive Poisson noise • Fractional stochastic process $\cdot$ Stochastic partial differential operator

Mathematics Subject Classifications (2010) $26 \mathrm{~A} 33$ • 60G22 • 35R60 • 34K37

\section{Introduction}

Define the Fourier transform $\mathcal{F} f$ (or $\hat{f}$ for brevity) of an integrable function $f$ on the $d$-dimensional Euclidean space $\mathbb{R}^{d}$ by

$$
\mathcal{F} f(\xi):=\int_{\mathbb{R}^{d}} e^{-i\langle\mathbf{x}, \xi\rangle} f(\mathbf{x}) d \mathbf{x},
$$

and extend the above definition to all tempered distributions as usual. Here we denote by $\langle\cdot, \cdot\rangle$ and $\|\cdot\|$ the standard inner product and norm on $\mathbb{R}^{d}$ respectively.

Let $\mathcal{S}:=\mathcal{S}\left(\mathbb{R}^{d}\right)$ be the space of all Schwartz functions on $\mathbb{R}^{d}$ and $\mathcal{S}^{\prime}:=$ $\mathcal{S}^{\prime}\left(\mathbb{R}^{d}\right)$ the space of all tempered distributions on $\mathbb{R}^{d}$. For $\gamma>0$, define the fractional Laplacian $(-\triangle)^{\gamma / 2}$ by

$$
\mathcal{F}\left((-\triangle)^{\gamma / 2} f\right)(\xi):=\|\xi\|^{\gamma} \mathcal{F} f(\xi), \quad f \in \mathcal{S} .
$$

The fractional Laplacian has the remarkable property of being dilationinvariant. It plays a crucial role in the definition of thin plate splines [4], is intimately tied to fractal stochastic processes (e.g., fractional Brownian fields) $[8,12]$ and stable Levy processes [3], and has been used in the study of singular obstacle problems $[2,10]$.

In this paper, we present a detailed mathematical investigation of the functional properties of dilation-invariant differential operators together with a characterization of their inverses. Our primary motivation is to provide a rigorous operator framework for solving the stochastic partial differential equation

$$
(-\triangle)^{\gamma / 2} \Phi=w
$$

with white noise $w$ as its driving term. We will show that this is feasible via the specification of a novel family of dilation-invariant left-inverses of the fractional Laplacian $(-\triangle)^{\gamma / 2}$ which have appropriate $L^{p}$-boundedness properties.

We say that a continuous linear operator $I$ from $\mathcal{S}$ to $\mathcal{S}^{\prime}$ is dilation-invariant if there exists a real number $\gamma$ such that

$$
I\left(\delta_{t} f\right)=t^{\gamma} \delta_{t}(I f) \text { for all } f \in \mathcal{S} \text { and } t>0,
$$

and translation-invariant if

$$
I\left(\tau_{\mathbf{x}_{0}} f\right)=\tau_{\mathbf{x}_{0}}(I f) \text { for all } f \in \mathcal{S} \text { and } \mathbf{x}_{0} \in \mathbb{R}^{d},
$$

where the dilation operator $\delta_{t}, t>0$ and the translation operator $\tau_{\mathbf{x}_{0}}, \mathbf{x}_{0} \in \mathbb{R}^{d}$ are defined by $\left(\delta_{t} f\right)(\mathbf{x})=f(t \mathbf{x})$ and $\tau_{\mathbf{x}_{0}} f(\mathbf{x})=f\left(\mathbf{x}-\mathbf{x}_{0}\right), f \in \mathcal{S}$, respectively. One 
may verify that the fractional Laplacian $(-\Delta)^{\gamma / 2}, \gamma>0$, is dilation-invariant and translation-invariant, a central property used in the definition of thin plate splines [4].

Next, we define the Riesz potential $I_{\gamma}[9]$ by

$$
I_{\gamma} f(\mathbf{x})=\pi^{-d / 2} 2^{-\gamma} \frac{\Gamma((d-\gamma) / 2)}{\Gamma(\gamma / 2)} \int_{\mathbb{R}^{d}}\|\mathbf{x}-\mathbf{y}\|^{\gamma-d} f(\mathbf{y}) d \mathbf{y}, \quad f \in \mathcal{S},
$$

where $0<\gamma<d$. Here the Gamma function $\Gamma$ is given by $\Gamma(z)=\int_{0}^{\infty} t^{z-1} e^{-t} d t$ when the real part $\operatorname{Re} z$ is positive, and is extended analytically to a meromorphic function on the complex plane. For any Schwartz function $f, I_{\gamma} f$ is continuous and satisfies

$$
\left|I_{\gamma} f(\mathbf{x})\right| \leq C_{\epsilon}\left(\sup _{\mathbf{z} \in \mathbb{R}^{d}}|f(\mathbf{z})|(1+\|\mathbf{z}\|)^{d+\epsilon}\right)(1+\|\mathbf{x}\|)^{\gamma-d} \quad \text { for all } \mathbf{x} \in \mathbb{R}^{d},
$$

where $\epsilon>0$ and $C_{\epsilon}$ is a positive constant, see also Theorem 2.1. Then the Riesz potential $I_{\gamma}$ is a continuous linear operator from $\mathcal{S}$ to $\mathcal{S}^{\prime}$. Moreover one may verify that $I_{\gamma}$ is dilation-invariant and translation-invariant, and also that $I_{\gamma}, 0<\gamma<d$, is the inverse of the fractional Laplacian $(-\triangle)^{\gamma / 2}$; i.e.,

$$
I_{\gamma}(-\triangle)^{\gamma / 2} f=(-\triangle)^{\gamma / 2} I_{\gamma} f=f \quad \text { for all } f \in \mathcal{S}
$$

because

$$
\mathcal{F}\left(I_{\gamma} f\right)(\xi)=\|\xi\|^{-\gamma} \mathcal{F} f(\xi), \quad f \in \mathcal{S}
$$

A natural question then is as follows:

Question 1 For any $\gamma>0$, is there a continuous linear operator $I$ from $\mathcal{S}$ to $\mathcal{S}^{\prime}$ that is translation-invariant and dilation-invariant, and that is an inverse of the fractional Laplacian $(-\triangle)^{\gamma / 2}$ ?

In the first result of this paper (Theorem 1.1), we give an affirmative answer to the above existence question for all positive non-integer numbers $\gamma$ with the invertibility replaced by the left-invertibility, and further prove the uniqueness of such a continuous linear operator.

To state that result, we recall some notation and definitions. Denote the dual pair between a Schwartz function and a tempered distribution using angle bracket $\langle\cdot, \cdot\rangle$, which is given by $\langle f, g\rangle=\int_{\mathbb{R}^{d}} f(\mathbf{x}) g(\mathbf{x}) d \mathbf{x}$ when $f, g \in \mathcal{S}$ (we remark that the dual pair between two complex-valued square-integrable functions is different from their standard inner product). A tempered distribution $f$ is said to be homogeneous of degree $\gamma$ if $\left\langle f, \delta_{t} g\right\rangle=t^{-\gamma-d}\langle f, g\rangle$ for all Schwartz functions $g$ and all positive numbers $t$. We notice that the multiplier $\|\xi\|^{-\gamma}$ in the Riesz potential $I_{\gamma}$, see (1.9), is a homogenous function of degree $-\gamma \in(-d, 0)$. This observation inspires us to follow the definition of 
homogeneous tempered distribution in [7] and then to extend the definition of the Riesz potential $I_{\gamma}$ to any non-integer number $\gamma>d$ as follows:

$$
\begin{aligned}
I_{\gamma} f(\mathbf{x}):= & \frac{(2 \pi)^{-d} \Gamma(d-\gamma)}{\Gamma\left(d+k_{0}-\gamma\right)} \int_{S^{d-1}} \int_{0}^{\infty} r^{k_{0}-\gamma+d-1} \\
& \times\left(-\frac{d}{d r}\right)^{k_{0}}\left(e^{i r\left\langle\mathbf{x}, \xi^{\prime}\right\rangle} \hat{f}\left(r \xi^{\prime}\right)\right) d r d \sigma\left(\xi^{\prime}\right), \quad f \in \mathcal{S},
\end{aligned}
$$

where $S^{n-1}=\left\{\xi^{\prime} \in \mathbb{R}^{d}:\left\|\xi^{\prime}\right\|=1\right\}$ is the unit sphere in $\mathbb{R}^{d}, d \sigma$ is the area element on $S^{n-1}$, and $k_{0}$ is a nonnegative integer larger than $\gamma-d$. Integration by parts shows that the above definition (1.10) of $I_{\gamma} f$ is independent on the nonnegative integer $k_{0}$ as long as it is larger than $\gamma-d$, and also that it coincides with the classical Riesz potential when $0<\gamma<d$ by letting $k_{0}=0$ and recalling that the inverse Fourier transform $\mathcal{F}^{-1} f$ of an integrable function $f$ is given by

$$
\mathcal{F}^{-1} f(\mathbf{x}):=(2 \pi)^{-d} \int_{\mathbb{R}^{d}} e^{i\langle\mathbf{x}, \xi\rangle} f(\xi) d \xi
$$

Because of the above consistency of definition, we call the continuous linear operator $I_{\gamma}, \gamma \in(0, \infty) \backslash\left(\mathbb{Z}_{+}+d\right)$, in (1.10) the generalized Riesz potential, where $\mathbb{Z}_{+}$is the set of all nonnegative integers.

Theorem 1.1 Let $\gamma$ be a positive number with $\gamma-d \notin \mathbb{Z}_{+}$, and let $I_{\gamma}$ be the linear operator defined by (1.10). Then $I_{\gamma}$ is the unique continuous linear operator from $\mathcal{S}$ to $\mathcal{S}^{\prime}$ that is dilation-invariant and translation-invariant, and that is a left inverse of the fractional Laplacian $(-\triangle)^{\gamma / 2}$.

Let $L^{p}:=L^{p}\left(\mathbb{R}^{d}\right), 1 \leq p \leq \infty$, be the space of all $p$-integrable functions on $\mathbb{R}^{d}$ with the standard norm $\|\cdot\|_{p}$. The Hardy-Littlewood-Sobolev fractional integration theorem [11] says that the Riesz potential $I_{\gamma}$ is a bounded linear operator from $L^{q}$ to $L^{p}$ when $1<p \leq \infty, 0<\gamma<d(1-1 / p)$ and $q=p d /(d+$ $\gamma p)$. Hence $I_{\gamma} f \in L^{p}$ for any Schwartz function $f$ when $0<\gamma<d(1-1 / p)$. We observe that for any non-integer number $\gamma$ larger than or equal to $d(1-$ $1 / p$ ), there exists a Schwartz function $f$ such that $I_{\gamma} f \notin L^{p}$, see Corollary 2.16. An implication of this negative result, which will become clearer in the sequel (cf. Section 4), is that we cannot generally use the translation-invariant inverse $I_{\gamma}$ to solve the stochastic partial differential equation (1.3). What is required instead is a special left-inverse of the fractional Laplacian that is dilationinvariant and $p$-integrable. Square-integrability in particular $(p=2)$ is a strict requirement when the driving noise is Gaussian and has been considered in prior work [12]; it leads to a fractional Brownian field solution, which is the multi-dimensional extension of Mandelbrot's celebrated fractional Brownian motion $[1,8]$. Our desire to extend this method of solution for non-Gaussian brands of noise leads to the second question. 
Question 2 Let $1 \leq p \leq \infty$ and $\gamma>0$. Is there a continuous linear operator I from $\mathcal{S}$ to $\mathcal{S}^{\prime}$ that is dilation-invariant and a left-inverse of the fractional Laplacian $(-\triangle)^{\gamma / 2}$ such that If $\in L^{p}$ for all Schwartz functions $f$ ?

In the second result of this paper (Theorem 1.2), we give an affirmative answer to the above question when both $\gamma$ and $\gamma-d(1-1 / p)$ are not integers, and show the uniqueness of such a continuous linear operator.

To state that result, we introduce some additional multi-integer notation. For $\mathbf{x}=\left(x_{1}, \ldots, x_{d}\right) \in \mathbb{R}^{d}$ and $\mathbf{j}=\left(j_{1}, \ldots, j_{d}\right) \in \mathbb{Z}_{+}^{d}$ (the $d$-copies of the set $\left.\mathbb{Z}_{+}\right)$, we set $|\mathbf{j}|:=\left|j_{1}\right|+\cdots+\left|j_{d}\right|, \mathbf{j} !:=j_{1} ! \cdots j_{d}$ ! with $0 !:=1, \mathbf{x}^{\mathbf{j}}:=x_{1}^{j_{1}} \cdots x_{d}^{j_{d}}$ and $\partial^{\mathbf{j}} f(\mathbf{x}):=\partial_{x_{1}}^{j_{1}} \cdots \partial_{x_{d}}^{j_{d}} f(\mathbf{x})$. For $1 \leq p \leq \infty$ and $\gamma>0$, we define the linear operator $I_{\gamma, p}$ from $\mathcal{S}$ to $\mathcal{S}^{\prime}$ with the help of the Fourier transform:

$$
\mathcal{F}\left(I_{\gamma, p} f\right)(\xi)=\left(\mathcal{F} f(\xi)-\sum_{|\mathbf{j}| \leq \gamma-d(1-1 / p)} \frac{\partial^{\mathbf{j}}(\mathcal{F} f)(\mathbf{0})}{\mathbf{j} !} \xi^{\mathbf{j}}\right)\|\xi\|^{-\gamma}, \quad f \in \mathcal{S},
$$

which is the natural $L^{p}$ extension of the fractional integral operator that was introduced in $[1,12,13]$ for $p=2$ and $\gamma \notin \mathbb{Z} / 2$.

We call $I_{\gamma, p}$ the p-integrable Riesz potential of degree $\gamma$, or the integrable Riesz potential for brevity. Indeed, when both $\gamma$ and $\gamma-d(1-1 / p)$ are nonintegers, the linear operator $I_{\gamma, p}$ is the unique left-inverse of the fractional Laplacian $(-\triangle)^{\gamma / 2}$ that enjoys the following dilation-invariance and stability properties.

Theorem 1.2 Let $1 \leq p \leq \infty$, and $\gamma$ is a positive number such that both $\gamma$ and $\gamma-d+d / p$ are not nonnegative integers. Then $I_{\gamma, p}$ in (1.12) is the unique dilation-invariant left-inverse of the fractional Laplacian $(-\triangle)^{\gamma / 2}$ such that its image of the $S$ chwartz space $\mathcal{S}$ is contained in $L^{p}$.

One of the primary application of the $p$-integrable Riesz potentials is the construction of generalized random processes by suitable functional integration of white noise [12-14]. These processes are defined by the stochastic partial differential equation (1.3), the motivation being that the solution should essentially display the same invariance properties as the defining operator (fractional Laplacian). In particular, these processes will exhibit some level of self-similarity (fractality) because $I_{\gamma, p}$ is dilation-invariant. However, they will in general not be stationary because the requirement for a stable inverse excludes translation invariance. It is this last aspect that deviates from the classical theory of stochastic processes and requires the type of mathematical safeguards that are provided in this paper. While the case of a white Gaussian noise excitation is fairly well understood [12], it is not yet so when the driving term is impulsive Poisson noise which leads to the specification of sparse stochastic processes with a finite rate of innovation. The current status has been to use the operator $I_{\gamma, 2}$ to specify sparse processes with the restriction that the impulse amplitude distribution must be symmetric [14, Theorem 2]. 
Our present contribution is to show that one can lift this restriction by considering the operator $I_{\gamma, 1}$, which is the proper inverse to handle general impulsive Poisson noise.

To state our third result, we recall some concepts about generalized random processes and Poisson noises. Let $\mathcal{D}$ be the space of all compactly supported $C^{\infty}$ functions with standard topology. A generalized random process is a random functional $\Phi$ defined on $\mathcal{D}$ (i.e., a random variable $\Phi(f)$ associated with every $f \in \mathcal{D}$ ) which is linear, continuous and compatible [6].

The white Poisson noise

$$
w(\mathbf{x}):=\sum_{k \in \mathbb{Z}} a_{k} \delta\left(\mathbf{x}-\mathbf{x}_{k}\right)
$$

is a generalized random process such that the random variable associated with a function $f \in \mathcal{D}$ is given by

$$
w(f):=\sum_{k \in \mathbb{Z}} a_{k} f\left(\mathbf{x}_{k}\right),
$$

where the $a_{k}$ 's are i.i.d. random variables with probability distribution $P(a)$, and where the $\mathbf{x}_{k}$ 's are random point locations in $\mathbb{R}^{n}$ which are mutually independent and follow a spatial Poisson distribution with Poisson parameter $\lambda>0$. The random point locations $\mathbf{x}_{k}$ in $\mathbb{R}^{n}$ follow a spatial Poisson distribution with Poisson parameter $\lambda>0$ meaning that for any measurable set $E$ with finite Lebesgue measure $|E|$, the probability of observing $n$ events in $E$ (i.e., the cardinality of the set $\left\{k \mid \mathbf{x}_{k} \in E\right\}$ is equal to $\left.n\right)$ is $\exp (-\lambda|E|)(\lambda|E|)^{n} / n !$. Thus, the Poisson parameter $\lambda$ represents the average number of random impulses per unit.

As the white Poisson noise $w$ is a generalized random process, the stochastic partial differential equation (1.3) can be interpreted as the following:

$$
\left\langle\Phi,(-\triangle)^{\gamma / 2} f\right\rangle=\langle w, f\rangle \text { for all } f \in \mathcal{D} .
$$

So if $I$ is a left-inverse of the fractional Laplacian operator $(-\Delta)^{\gamma / 2}$, then

$$
\Phi=I^{*} w
$$

is literally the solution of the stochastic partial differential equation (1.3) as

$$
\left\langle I^{*} w,(-\triangle)^{\gamma / 2} f\right\rangle=\left\langle w, I(-\Delta)^{\gamma / 2} f\right\rangle=\langle w, f\rangle \quad \text { for all } f \in \mathcal{D},
$$

where $I^{*}$ is the conjugate operator of the continuous linear operator $I$ from $\mathcal{S}$ to $\mathcal{S}^{\prime}$ defined by

$$
\left\langle I^{*} f, g\right\rangle:=\langle f, I g\rangle \quad \text { for all } f, g \in \mathcal{S} .
$$

The above observation is usable only if we can specify a left-inverse (or equivalently we can impose appropriate boundary condition) so that $I^{*} w$ defines a bona fide generalized random process in the sense of Gelfand and 
Vilenkin; mathematically, the latter is equivalent to providing its characteristic functional by the Minlos-Bochner Theorem (cf. Section 4). The following result establishes that $P_{\gamma} w:=I_{\gamma, 1}^{*} w$ is a proper solution of the stochastic partial differential equation (1.3), where $w$ is the Poisson noise defined by (1.13).

Theorem 1.3 Let $\gamma$ be a positive non-integer number, $\lambda$ be a positive number, $P(a)$ be a probability distribution with $\int_{\mathbb{R}}|a| d P(a)<\infty$, and $I_{\gamma, 1}$ be defined as in (1.12). For any $f \in \mathcal{D}$, define the random variable $P_{\gamma} w$ associated with $f$ by

$$
P_{\gamma} w(f):=\sum_{k} a_{k} I_{\gamma, 1}(f)\left(\mathbf{x}_{k}\right)
$$

where the $a_{k}$ 's are i.i.d. random variables with probability distribution $P(a)$, and the $\mathbf{x}_{k}$ 's are random point locations in $\mathbb{R}^{n}$ which are mutually independent and follow a spatial Poisson distribution with Poisson parameter $\lambda$. Then $P_{\gamma} w$ is the generalized random process associated with the characteristic functional

$$
\mathcal{Z}_{P_{\gamma} w}(f)=\exp \left(\lambda \int_{\mathbb{R}^{d}} \int_{\mathbb{R}}\left(e^{-i a\left(I_{\gamma, 1} f\right)(\mathbf{x})}-1\right) d P(a) d \mathbf{x}\right), \quad f \in \mathcal{D} .
$$

The organization of the paper is as follows. In Section 2, we first introduce a linear operator $J_{\Omega}$ for any homogeneous function $\Omega \in C^{\infty}\left(\mathbb{R}^{d} \backslash\{\boldsymbol{0}\}\right)$ of degree $-\gamma$, where $\gamma-d \notin \mathbb{Z}_{+}$. The linear operator $J_{\Omega}$ becomes the generalized Riesz potential $I_{\gamma}$ in (1.10) when $\Omega(\xi)=\|\xi\|^{-\gamma}$; conversely, any derivative of the generalized Riesz potential $I_{\gamma}$ is a linear operator $J_{\Omega}$ associated with some homogeneous function $\Omega$ :

$$
\partial^{\mathbf{j}} I_{\gamma} f=J_{\Omega_{\mathbf{j}}} f \quad \text { for all } f \in \mathcal{S} \text { and } \mathbf{j} \in \mathbb{Z}_{+}^{d},
$$

where $\Omega_{\mathbf{j}}(\xi)=(i \xi)^{\mathbf{j}}\|\xi\|^{-\gamma}$. We then study various properties of the above linear operator $J_{\Omega}$, such as polynomial decay property, dilation-invariance, translation-invariance, left-invertibility, and non-integrability in the spatial domain and in the Fourier domain. The proof of Theorem 1.1 is given at the end of Section 2.

In Section 3, we introduce a linear operator $U_{\Omega, p}$ for any homogeneous function $\Omega \in C^{\infty}\left(\mathbb{R}^{d} \backslash\{\boldsymbol{0}\}\right)$ of degree $-\gamma$, where $1 \leq p \leq \infty$. The above linear operator $U_{\Omega, p}$ becomes the operator $I_{\gamma, p}$ in (1.12) when $\Omega(\xi)=\|\xi\|^{-\gamma}$, and the operator $J_{\Omega}$ in (2.1) when $0<\gamma<d(1-1 / p)$. We show that the linear operator $U_{\Omega, p}$ is dilation-invariant, translation-variant and $p$-integrable, and is a left-inverse of the fractional Laplacian $(-\triangle)^{\gamma / 2}$ when $\Omega(\xi)=\|\xi\|^{-\gamma}$. The proof of Theorem 1.2 is given at the end of Section 3.

In Section 4, we give the proof of Theorem 1.3 and show that the generalized random process $P_{\gamma} w$ can be evaluated pointwise in the sense that we can replace the function $f$ in (1.18) by the delta functional $\delta$. 
In this paper, the capital letter $C$ denotes an absolute positive constant which may vary depending on the occurrence.

\section{Generalized Riesz potentials}

Let $\gamma$ be a real number such that $\gamma-d \notin \mathbb{Z}_{+}$, and let $\Omega \in C^{\infty}\left(\mathbb{R}^{d} \backslash\{\boldsymbol{0}\}\right)$ be a homogeneous function of degree $-\gamma$. Following the definition of homogenous tempered distributions in [7], we define the linear operator $J_{\Omega}$ from $\mathcal{S}$ to $\mathcal{S}^{\prime}$ by

$$
\begin{aligned}
J_{\Omega} f(\mathbf{x}):= & \frac{(2 \pi)^{-d} \Gamma(d-\gamma)}{\Gamma\left(d+k_{0}-\gamma\right)} \int_{S^{d-1}} \int_{0}^{\infty} \Omega\left(\xi^{\prime}\right) r^{k_{0}-\gamma+d-1} \\
& \times\left(-\frac{d}{d r}\right)^{k_{0}}\left(e^{i r\left\langle\mathbf{x}, \xi^{\prime}\right\rangle} \hat{f}\left(r \xi^{\prime}\right)\right) d r d \sigma\left(\xi^{\prime}\right), \quad f \in \mathcal{S},
\end{aligned}
$$

where $S^{n-1}=\left\{\xi^{\prime} \in \mathbb{R}^{d}:\left\|\xi^{\prime}\right\|=1\right\}$ is the unit sphere in $\mathbb{R}^{d}, d \sigma$ is the area element on $S^{n-1}$, and $k_{0}$ is a nonnegative integer larger than $\gamma-d$.

Note that the linear operator $J_{\Omega}$ in (2.1) becomes the generalized Riesz potential $I_{\gamma}$ in (1.10) when $\Omega(\xi)=\|\xi\|^{-\gamma}$ and $\gamma>0$. Therefore we call the linear operator $J_{\Omega}$ in (2.1) the generalized Riesz potential associated with the homogeneous function $\Omega$ of degree $-\gamma$, or the generalized Riesz potential for brevity.

The above definition of the generalized Riesz potential $J_{\Omega}$ is independent on the nonnegative integer $k_{0}$ as long as it satisfies $k_{0}>\gamma-d$, that can be shown by integration by parts. Then, for $\gamma \in(-\infty, d)$, we may take $k_{0}=0$ and reformulate (2.1) as follows:

$$
J_{\Omega} f(\mathbf{x})=(2 \pi)^{-d} \int_{\mathbb{R}^{d}} e^{i\langle\mathbf{x}, \xi\rangle} \Omega(\xi) \hat{f}(\xi) d \xi \quad \text { for all } f \in \mathcal{S},
$$

or equivalently

$$
\widehat{J_{\Omega} f}(\xi)=\Omega(\xi) \hat{f}(\xi) \text { for all } f \in \mathcal{S}
$$

so that the role of the homogeneous function $\Omega(\xi)$ in (2.1) is essentially that of the Fourier symbol for a conventional translation-invariant operator.

Let $\mathcal{S}_{\infty}$ be the space of all Schwartz functions $f$ such that $\partial^{\mathbf{i}} \hat{f}(\mathbf{0})=0$ for all $\mathbf{i} \in \mathbb{Z}_{+}^{d}$, or equivalently that $\int_{\mathbb{R}^{d}} \mathbf{x}^{\mathbf{j}} f(\mathbf{x}) d \mathbf{x}=0$ for all $\mathbf{j} \in \mathbb{Z}_{+}^{d}$. Given a homogenous function $\Omega \in C^{\infty}\left(\mathbb{R}^{d} \backslash\{\mathbf{0}\}\right)$, define the linear operator $i_{\Omega}$ on $\mathcal{S}_{\infty}$ by

$$
\widehat{i_{\Omega} f}(\xi)=\Omega(\xi) \hat{f}(\xi), \quad f \in \mathcal{S}_{\infty} .
$$


Clearly $i_{\Omega}$ is a continuous linear operator on the closed linear subspace $\mathcal{S}_{\infty}$ of $\mathcal{S}$. For any function $f \in \mathcal{S}_{\infty}$, applying the integration-by-parts technique $k_{0}$ times and noticing that $\lim _{\epsilon \rightarrow 0} \epsilon^{-\gamma}\left|\partial^{\mathbf{i}} \hat{f}\left(\epsilon \xi^{\prime}\right)\right|=0$ for all $\xi^{\prime} \in S^{d-1}$ and $\mathbf{i} \in \mathbb{Z}_{+}^{d}$, we obtain that

$$
\begin{aligned}
J_{\Omega} f(\mathbf{x})= & \frac{(2 \pi)^{-d} \Gamma(d-\gamma)}{\Gamma\left(d+k_{0}-\gamma\right)} \lim _{\epsilon \rightarrow 0} \int_{S^{d-1}} \int_{\epsilon}^{\infty} r^{k_{0}+d-\gamma-1} \Omega\left(\xi^{\prime}\right) \\
& \times\left(-\frac{d}{d r}\right)^{k_{0}}\left(e^{i r\left\langle\mathbf{x}, \xi^{\prime}\right\rangle} \hat{f}\left(r \xi^{\prime}\right)\right) d r d \sigma\left(\xi^{\prime}\right) \\
= & (2 \pi)^{-d} \lim _{\epsilon \rightarrow 0} \int_{S^{d-1}} \int_{\epsilon}^{\infty} \Omega\left(\xi^{\prime}\right) r^{d-\gamma-1} e^{i r\left\langle\mathbf{x}, \xi^{\prime}\right\rangle} \hat{f}\left(r \xi^{\prime}\right) d r d \sigma\left(\xi^{\prime}\right) \\
= & (2 \pi)^{-d} \int_{\mathbb{R}^{d}} e^{i\langle\mathbf{x}, \xi\rangle} \Omega(\xi) \hat{f}(\xi) d \xi=i_{\Omega} f(\mathbf{x}) .
\end{aligned}
$$

Hence the generalized Riesz potential $J_{\Omega}$ is the extension of the linear operator $i_{\Omega}$ from the closed subspace $\mathcal{S}_{\infty}$ to the whole space $\mathcal{S}$.

In the sequel, we will study further properties of the generalized Riesz potential $J_{\Omega}$, such as the polynomial decay property (Theorem 2.1), the continuity as a linear operator from $\mathcal{S}$ to $\mathcal{S}^{\prime}$ (Corollary 2.3), the translationinvariance and dilation-invariance (Theorem 2.7), the composition and leftinverse property (Theorem 2.8 and Corollary 2.9), the uniqueness of various extensions of the linear operator $i_{\Omega}$ from the closed subspace $\mathcal{S}_{\infty}$ to the whole space $\mathcal{S}$ (Theorems 2.11 and 2.14), the non-integrability in the spatial domain (Theorem 2.15), and the non-integrability in the Fourier domain (Theorem 2.17). Some of those properties will be used to prove Theorem 1.1, which is included at the end of this section.

\subsection{Polynomial decay property and continuity}

Theorem 2.1 Let $\gamma$ be a positive number with $\gamma-d \notin \mathbb{Z}_{+}, k_{0}$ be the smallest nonnegative integer larger than $\gamma-d$, and let $\Omega \in C^{\infty}\left(\mathbb{R}^{d} \backslash\{\mathbf{0}\}\right)$ be a homogeneous function of degree $-\gamma$. If there exist positive constants $\epsilon$ and $C_{\epsilon}$ such that

$$
|f(\mathbf{x})| \leq C_{\epsilon}(1+\|\mathbf{x}\|)^{-k_{0}-d-\epsilon} \text { for all } \mathbf{x} \in \mathbb{R}^{d},
$$

then there exists a positive constant $C$ such that

$$
\left|J_{\Omega} f(\mathbf{x})\right| \leq C\left(\sup _{\mathbf{z} \in \mathbb{R}^{d}}|f(\mathbf{z})|(1+\|\mathbf{z}\|)^{k_{0}+d+\epsilon}\right)(1+\|\mathbf{x}\|)^{\gamma-d}, \quad \mathbf{x} \in \mathbb{R}^{d} .
$$


Proof Noting that $\left(\frac{d}{d r}\right)^{s} e^{i r\left\langle\mathbf{x}, \xi^{\prime}\right\rangle}=s !\left(\sum_{|\mathbf{i}|=s} \frac{(i \mathbf{x})^{\mathbf{i}} \xi^{\mathbf{i}}}{\mathbf{i} !}\right) e^{i r\left\langle\mathbf{x}, \xi^{\prime}\right\rangle}$ and $\left(\frac{d}{d r}\right)^{k_{0}-s} \hat{f}\left(r \xi^{\prime}\right)=$ $\left(k_{0}-s\right) ! \sum_{|\mathbf{j}|=k_{0}-s} \frac{\left(\xi^{\prime}\right)^{\mathbf{j}} \partial^{\mathbf{j}} \hat{f}\left(r \xi^{\prime}\right)}{\mathbf{j} !}$ for all $0 \leq s \leq k_{0}$, we obtain from the Leibniz rule that

$$
\begin{aligned}
\left(\frac{d}{d r}\right)^{k_{0}}\left(e^{i r\left\langle\mathbf{x}, \xi^{\prime}\right\rangle} \hat{f}\left(r \xi^{\prime}\right)\right) & =\sum_{s=0}^{k_{0}}\left(\begin{array}{c}
k_{0} \\
s
\end{array}\right)\left\{\left(\frac{d}{d r}\right)^{k_{0}-s} e^{i r\left\langle\mathbf{x}, \xi^{\prime}\right\rangle}\right\} \cdot\left\{\left(\frac{d}{d r}\right)^{s} \hat{f}\left(r \xi^{\prime}\right)\right\} \\
& =\left(\sum_{|\mathbf{i}|+|\mathbf{j}|=k_{0}} \frac{k_{0} !}{\mathbf{i} ! \mathbf{j} !}(i \mathbf{x})^{\mathbf{i}}\left(\xi^{\prime}\right)^{\mathbf{i}+\mathbf{j}} \partial^{\mathbf{j}} \hat{f}\left(r \xi^{\prime}\right)\right) e^{i r\left\langle\mathbf{x}, \xi^{\prime}\right\rangle} .
\end{aligned}
$$

Substituting the above expression into (2.1) we get

$$
\begin{aligned}
J_{\Omega} f(\mathbf{x})= & (-1)^{k_{0}} \sum_{|\mathbf{i}|+|\mathbf{j}|=k_{0}} \frac{k_{0} !}{\mathbf{i} ! \mathbf{j} !}(i \mathbf{x})^{\mathbf{i}}\left\{\frac{(2 \pi)^{-d} \Gamma(d-\gamma)}{\Gamma\left(d+k_{0}-\gamma\right)}\right. \\
& \left.\times \int_{\mathbb{R}^{d}} e^{i\langle\mathbf{x}, \xi\rangle}\left(\xi^{\mathbf{i}+\mathbf{j}} \Omega(\xi)\right) \partial^{\mathbf{j}} \hat{f}(\xi) d \xi\right\} \\
= & \frac{\Gamma(d-\gamma)}{\Gamma\left(d+k_{0}-\gamma\right)} \sum_{|\mathbf{i}|+|\mathbf{j}|=k_{0}} \frac{k_{0} !}{\mathbf{i} ! \mathbf{j} !}(-\mathbf{x})^{\mathbf{i}} J_{\Omega_{\mathbf{i}+\mathbf{j}}}\left(f_{\mathbf{j}}\right)(\mathbf{x}),
\end{aligned}
$$

where $\Omega_{\mathbf{i}+\mathbf{j}}(\xi)=(i \xi)^{\mathbf{i}+\mathbf{j}} \Omega(\xi)$ and $f_{\mathbf{j}}(\mathbf{x})=\mathbf{x}^{\mathbf{j}} f(\mathbf{x})$. Denote the inverse Fourier transform of $\Omega_{\mathbf{k}},|\mathbf{k}|=k_{0}$, by $K_{\mathbf{k}}$. Then $K_{\mathbf{k}} \in C^{\infty}\left(\mathbb{R}^{d} \backslash\{\mathbf{0}\}\right)$ is a homogeneous function of degree $\gamma-k_{0}-d$ [7, Theorems 7.1.16 and 7.1.18], and hence there exists a positive constant $C$ such that

$$
\left|K_{\mathbf{k}}(\mathbf{x})\right| \leq C\|\mathbf{x}\|^{\gamma-k_{0}-d} \quad \text { for all } \mathbf{x} \in \mathbb{R}^{d} \backslash\{\mathbf{0}\} .
$$

For any $\epsilon>0$ and $\beta \in(0, d)$, we have

$$
\begin{aligned}
\int_{\mathbb{R}^{d}} & \|\mathbf{x}-\mathbf{y}\|^{-\beta}(1+\|\mathbf{y}\|)^{-d-\epsilon} d \mathbf{y} \\
\leq & \left(\int_{\|\mathbf{y}\| \leq(\|\mathbf{x}\|+1) / 2}+\int_{(\|\mathbf{x}\|+1) / 2 \leq\|\mathbf{y}\| \leq 2(\|\mathbf{x}\|+1)}+\int_{\|\mathbf{y}\| \geq 2(\|\mathbf{x}\|+1)}\right) \\
& \times\|\mathbf{x}-\mathbf{y}\|^{-\beta}(1+\|\mathbf{y}\|)^{-d-\epsilon} d \mathbf{y} \\
\leq & C(1+\|\mathbf{x}\|)^{-\beta} .
\end{aligned}
$$

Combining (2.8), (2.9) and (2.10) yields

$$
\begin{aligned}
\left|J_{\Omega} f(\mathbf{x})\right| & \leq C \sum_{|\mathbf{i}|+|\mathbf{j}|=k_{0}}|\mathbf{x}|^{|\mathbf{i}|}\left|\int_{\mathbb{R}^{d}} K_{\mathbf{i}+\mathbf{j}}(\mathbf{x}-\mathbf{y}) \mathbf{y}^{\mathbf{j}} f(\mathbf{y})\right| d \mathbf{y} \\
& \leq C(1+\|\mathbf{x}\|)^{k_{0}} \int_{\mathbb{R}^{d}}\|\mathbf{x}-\mathbf{y}\|^{\gamma-k_{0}-d}(1+\|\mathbf{y}\|)^{k_{0}}|f(\mathbf{y})| d \mathbf{y} \\
& \leq C\left(\sup _{\mathbf{z} \in \mathbb{R}^{d}}|f(\mathbf{z})|(1+\|\mathbf{z}\|)^{k_{0}+d+\epsilon}\right)(1+\|\mathbf{x}\|)^{\gamma-d} .
\end{aligned}
$$

This proves the desired polynomial decay estimate (2.7). 
For any $f \in \mathcal{S}$ and $\mathbf{j} \in \mathbb{Z}_{+}^{d}$ with $|\mathbf{j}|=1$, it follows from (2.1) that

$$
\begin{aligned}
\partial^{\mathbf{j}}\left(J_{\Omega} f\right)(\mathbf{x})= & J_{\Omega}\left(\partial^{\mathbf{j}} f\right)(\mathbf{x}) \\
= & \frac{(2 \pi)^{-d} \Gamma(d-\gamma)}{\Gamma\left(d+k_{0}-\gamma\right)} \int_{S^{d-1}} \int_{0}^{\infty} \Omega\left(\xi^{\prime}\right)\left(i \xi^{\prime}\right)^{\mathbf{j}} r^{k_{0}+d-\gamma-1} \\
& \times\left(-\frac{d}{d r}\right)^{k_{0}}\left(e^{i r\left\langle\mathbf{x}, \xi^{\prime}\right\rangle} \hat{f}\left(r \xi^{\prime}\right) r\right) d r d \sigma\left(\xi^{\prime}\right) \\
= & \frac{(2 \pi)^{-d} \Gamma(d-\gamma)}{\Gamma\left(d+k_{0}-\gamma\right)} \int_{S^{d-1}} \int_{0}^{\infty} \Omega\left(\xi^{\prime}\right)\left(i \xi^{\prime}\right)^{\mathbf{j}} r^{k_{0}+d-\gamma-1} \\
& \times\left\{r\left(-\frac{d}{d r}\right)^{k_{0}}\left(e^{i r\left\langle\mathbf{x}, \xi^{\prime}\right\rangle} \hat{f}\left(r \xi^{\prime}\right)\right)\right. \\
& \left.-k_{0}\left(-\frac{d}{d r}\right)^{k_{0}-1}\left(e^{i r\left\langle\mathbf{x}, \xi^{\prime}\right\rangle} \hat{f}\left(r \xi^{\prime}\right)\right)\right\} d r d \sigma\left(\xi^{\prime}\right) \\
= & \left(\frac{d+k_{0}-\gamma}{d-\gamma}-k_{0} \frac{1}{d-\gamma}\right) J_{\Omega_{\mathbf{j}}} f(\mathbf{x})=J_{\Omega_{\mathbf{j}}} f(\mathbf{x}),
\end{aligned}
$$

where $\Omega_{\mathbf{j}}(\xi)=(i \xi)^{\mathbf{j}} \Omega(\xi)$. Applying the argument inductively leads to

$$
\partial^{\mathbf{j}}\left(J_{\Omega} f\right)=J_{\Omega}\left(\partial^{\mathbf{j}} f\right)=J_{\Omega_{\mathbf{j}}} f \quad \text { for all } f \in \mathcal{S} \text { and } \mathbf{j} \in \mathbb{Z}_{+}^{d},
$$

where $\Omega_{\mathbf{j}}(\xi)=(i \xi)^{\mathbf{j}} \Omega(\xi)$. This together with Theorem 2.1 shows that $J_{\Omega} f$ is a smooth function on $\mathbb{R}^{d}$ for any Schwartz function $f$.

Corollary 2.2 Let $\gamma, k_{0}$ and $\Omega$ be as in Theorem 2.1. If $f$ satisfies (2.6) for some positive constants $\epsilon$ and $C_{\epsilon}$, then for any $\mathbf{j} \in \mathbb{Z}_{+}^{d}$ with $|\mathbf{j}|<\gamma$ there exists a positive constant $C_{\mathbf{j}}$ such that

$$
\left|\partial^{\mathbf{j}}\left(J_{\Omega} f\right)(\mathbf{x})\right| \leq C_{\mathbf{j}}\left(\sup _{\mathbf{z} \in \mathbb{R}^{d}}|f(\mathbf{z})|(1+\|\mathbf{z}\|)^{k_{0}+d+\epsilon}\right)(1+\|\mathbf{x}\|)^{\gamma-|\mathbf{j}|-d}, \mathbf{x} \in \mathbb{R}^{d} .
$$

An easy consequence of the above smoothness result about $J_{\Omega} f$ is the continuity of the generalized Riesz potential $J_{\Omega}$ from $\mathcal{S}$ to $\mathcal{S}^{\prime}$.

Corollary 2.3 Let $\gamma$ be a positive number with $\gamma-d \notin \mathbb{Z}_{+}$, and let $\Omega \in$ $C^{\infty}\left(\mathbb{R}^{d} \backslash\{\mathbf{0}\}\right)$ be a homogeneous function of degree $-\gamma$. Then the generalized Riesz potential $J_{\Omega}$ associated with the homogeneous function $\Omega$ is a continuous linear operator from $\mathcal{S}$ to $\mathcal{S}^{\prime}$.

Now consider the generalized Riesz potential $J_{\Omega}$ when $\Omega$ is a homogeneous function of positive degree $\alpha$. In this case,

$$
J_{\Omega} f(\mathbf{x})=(2 \pi)^{-d} \int_{\mathbb{R}^{d}} e^{i\langle\mathbf{x}, \xi\rangle} \Omega(\xi) \hat{f}(\xi) d \xi \quad \text { for all } f \in \mathcal{S}
$$


by (2.2). Applying the integration-by-parts technique then gives

$$
J_{\Omega} f(\mathbf{x})=(2 \pi)^{-d}\left(-i \mathbf{x}^{\mathbf{i}}\right)^{-1} \sum_{\mathbf{j}+\mathbf{k}=\mathbf{i}} \frac{\mathbf{i} !}{\mathbf{j} ! \mathbf{k} !} \int_{\mathbb{R}^{d}} e^{i\langle\mathbf{x}, \xi\rangle} \partial^{\mathbf{j}} \Omega(\xi) \partial^{\mathbf{k}} \hat{f}(\xi) d \xi
$$

for any $\mathbf{i} \in \mathbb{Z}_{+}^{d}$. This, together with the identity

$$
1=\sum_{|\mathbf{I}|=\lceil\alpha\rceil-|\mathbf{j}|} \frac{(\lceil\alpha\rceil-|\mathbf{j}|) !}{\mathbf{l} !}\left(\frac{i \xi}{\|\xi\|^{2}}\right)^{\mathbf{l}}(-i \xi)^{\mathbf{l}}, \quad|\mathbf{j}| \leq\lceil\alpha\rceil,
$$

leads to the following estimate of $J_{\Omega} f(\mathbf{x})$ :

$$
\begin{aligned}
\left|J_{\Omega} f(\mathbf{x})\right| & \leq C(1+\|\mathbf{x}\|)^{-\lceil\alpha\rceil} \sum_{|\mathbf{j}|+|\mathbf{k}| \leq\lceil\alpha\rceil,|\mathbf{I}|=\lceil\alpha\rceil-|\mathbf{j}|}\left|\int_{\mathbb{R}^{d}} e^{i|\mathbf{x}, \xi\rangle} \Omega_{\mathbf{j}, \mathbf{I}}(\xi) \xi^{\mathbf{l}} \partial^{\mathbf{k}} \hat{f}(\xi) d \xi\right| \\
& \leq C(1+\|\mathbf{x}\|)^{-\lceil\alpha\rceil} \sum_{|\mathbf{j}|+|\mathbf{k}| \leq\lceil\alpha\rceil,|\mathbf{I}|+|\mathbf{j}|=\lceil\alpha\rceil}\left|I_{\Omega_{\mathbf{j}, \mathbf{l}}} f_{\mathbf{l}, \mathbf{k}}(\mathbf{x})\right|,
\end{aligned}
$$

where $\lceil\alpha\rceil$ is the smallest integer larger than $\alpha, \Omega_{\mathbf{j}, \mathbf{l}}(\xi)=\partial^{\mathbf{j}} \Omega(\xi)\left(i \xi /\|\xi\|^{2}\right)^{\mathbf{I}}$, and $\widehat{f_{\mathbf{l}, \mathbf{k}}}(\xi)=(-i \xi)^{\mathbf{l}} \partial^{\mathbf{k}} \hat{f}(\xi)$. Note that $\Omega_{\mathbf{j}, \mathbf{l}} \in C^{\infty}\left(\mathbb{R}^{d} \backslash\{\mathbf{0}\}\right)$ is a homogeneous function of degree $\alpha-\lceil\alpha\rceil<0$ when $|\mathbf{j}|+|\mathbf{I}|=\lceil\alpha\rceil$, and also that functions $f_{\mathbf{l}, \mathbf{k}}(\mathbf{x}),|\mathbf{k}|,|\mathbf{I}| \leq\lceil\alpha\rceil$ are linear combinations of $\mathbf{x}^{\mathbf{i}} \partial^{\mathbf{j}} f(\mathbf{x}),|\mathbf{i}|,|\mathbf{j}| \leq\lceil\alpha\rceil$. We then apply Theorem 2.1 to obtain the following polynomial decay estimate of $J_{\Omega} f$ when $\Omega$ is a homogeneous function of positive degree:

Proposition 2.4 Let $\alpha$ be a positive non-integer number, and $\Omega \in C^{\infty}\left(\mathbb{R}^{d} \backslash\{\boldsymbol{0}\}\right)$ be a homogeneous function of degree $\alpha$. If there exist positive constants $\epsilon$ and $C_{\epsilon}$ such that

$$
\sum_{|\mathbf{i}| \leq\lceil\alpha\rceil}\left|\partial^{\mathbf{i}} f(\mathbf{x})\right| \leq C_{\epsilon}(1+\|\mathbf{x}\|)^{-\lceil\alpha\rceil-d-\epsilon} \text { for all } \mathbf{x} \in \mathbb{R}^{d},
$$

then there exists a positive constant $C$ such that

$$
\left|J_{\Omega} f(\mathbf{x})\right| \leq C\left(\sum_{|\mathbf{i}| \leq\lceil\alpha\rceil} \sup _{\mathbf{z} \in \mathbb{R}^{d}}\left|\partial^{\mathbf{i}} f(\mathbf{z})\right|(1+\|\mathbf{z}\|)^{\lceil\alpha\rceil+d+\epsilon}\right)(1+\|\mathbf{x}\|)^{-\alpha-d}
$$

for all $\mathbf{x} \in \mathbb{R}^{d}$

The estimates in (2.7) and (2.13) indicate that the generalized Riesz potential $J_{\Omega} f$ has faster polynomial decay at infinity when the degree of the homogeneous function $\Omega$ becomes larger. Next, we show that the generalized Riesz potential $J_{\Omega} f$ has faster polynomial decay at infinity when $f$ has vanishing moments up to some order; i.e.,

$$
\int_{\mathbb{R}^{d}} \mathbf{x}^{\mathbf{i}} f(\mathbf{x}) d \mathbf{x}=0,|\mathbf{i}| \leq m_{0}
$$


where $m_{0} \geq 0$. In this case, $\partial^{\mathbf{i}} \hat{f}(\mathbf{0})=0$ for all $|\mathbf{i}| \leq m_{0}$, and hence

$$
\hat{f}(\xi)=\sum_{|\mathbf{k}|=m_{0}+1} \frac{m_{0}+1}{\mathbf{k} !} \int_{0}^{1} \xi^{\mathbf{k}} \partial^{\mathbf{k}} \hat{f}(t \xi)(1-t)^{m_{0}} d t
$$

by the Taylor expansion to $\hat{f}$ at the origin. Now we assume that $\Omega \in$ $C^{\infty}\left(\mathbb{R}^{d} \backslash\{\boldsymbol{0}\}\right)$ is a homogeneous function of degree $\alpha \in\left(-m_{0}-1, \infty\right) \backslash \mathbb{Z}$. Then

$$
\begin{aligned}
\left|J_{\Omega} f(\mathbf{x})\right| & \leq C \sum_{|\mathbf{k}|=m_{0}+1} \int_{0}^{1} \int_{\|\xi\| \leq 1}|\xi|^{\alpha+m_{0}+1}\left|\partial^{\mathbf{k}} \hat{f}(t \xi)\right| d \xi d t+C \int_{|\xi| \geq 1}|\xi|^{\alpha}|\hat{f}(\xi)| d \xi \\
& \leq C \sum_{|\mathbf{i}| \leq m_{0}+1} \sup _{\xi \in \mathbb{R}^{d}}\left((1+\|\xi\|)^{\lceil\alpha\rceil+d}\left|\partial^{\mathbf{i}} \hat{f}(\xi)\right|\right)
\end{aligned}
$$

for all $\mathbf{x} \in \mathbb{R}^{d}$ with $\|\mathbf{x}\| \leq 1$, and

$$
\begin{aligned}
& \left|J_{\Omega} f(\mathbf{x})\right| \leq C \sum_{|\mathbf{k}|=m_{0}+1} \int_{0}^{1}\left|\int_{\mathbb{R}^{d}} e^{-i\langle\mathbf{x}, \xi\rangle} \phi(\|\mathbf{x}\| \xi) \xi^{\mathbf{k}} \Omega(\xi) \partial^{\mathbf{k}} \hat{f}(t \xi) d \xi\right| d t \\
& +C \sum_{|\mathbf{k}|=m_{0}+1} \int_{0}^{1}\left|\int_{\mathbb{R}^{d}} e^{-i\langle\mathbf{x}, \xi\rangle}(\phi(\xi)-\phi(\|\mathbf{x}\| \xi)) \xi^{\mathbf{k}} \Omega(\xi) \partial^{\mathbf{k}} \hat{f}(t \xi) d \xi\right| d t \\
& +C\left|\int_{\mathbb{R}^{d}} e^{-i\langle\mathbf{x}, \xi\rangle}(1-\phi(\xi)) \Omega(\xi) \hat{f}(\xi) d \xi\right| \\
& \leq C(1+\|\mathbf{x}\|)^{-\lceil\alpha\rceil-m_{0}-d}\left\{\sum_{|\mathbf{k}|=m_{0}+1,|\mathbf{j}| \leq\lceil\alpha\rceil+m_{0}+d}\right. \\
& \left.\times \int_{0}^{1} \int_{\mathbb{R}^{d}}\left|\partial^{\mathbf{j}}\left(\phi(\|\mathbf{x}\| \xi) \xi^{\mathbf{k}} \Omega(\xi) \partial^{\mathbf{k}} \hat{f}(t \xi)\right)\right| d \xi d t\right\} \\
& +C(1+\|\mathbf{x}\|)^{-\lceil\alpha\rceil-m_{0}-d-1}\left\{\sum_{|\mathbf{k}|=m_{0}+1,|\mathbf{j}| \leq\lceil\alpha\rceil+m_{0}+d+1}\right. \\
& \left.\times \int_{0}^{1} \int_{\mathbb{R}^{d}}\left|\partial^{\mathbf{j}}\left((\phi(\xi)-\phi(\|\mathbf{x}\| \xi)) \xi^{\mathbf{k}} \Omega(\xi) \partial^{\mathbf{k}} \hat{f}(t \xi)\right)\right| d \xi d t\right\} \\
& +C(1+\|\mathbf{x}\|)^{-\lceil\alpha\rceil-m_{0}-d-1} \\
& \times\left\{\sum_{|\mathbf{j}| \leq\lceil\alpha\rceil+m_{0}+d+1} \int_{\mathbb{R}^{d}}\left|\partial^{\mathbf{j}}((1-\phi(\xi)) \Omega(\xi) \hat{f}(\xi))\right| d \xi\right\} \\
& \leq C\left(\sum_{|\mathbf{i}| \leq\lceil\alpha\rceil+2 m_{0}+d+2} \sup _{\xi \in \mathbb{R}^{d}}(1+\|\xi\|)^{\lceil\alpha\rceil+d}\left|\partial^{\mathbf{i}} \hat{f}(\xi)\right|\right)(1+\|\mathbf{x}\|)^{-\alpha-m_{0}-d-1}
\end{aligned}
$$

for all $\mathbf{x} \in \mathbb{R}^{d}$ with $\|\mathbf{x}\| \geq 1$, where $\phi$ is a $C^{\infty}$ function such that $\phi(\xi)=1$ for all $\xi$ in the unit ball $B(\mathbf{0}, 1)$ centered at the origin, and $\phi(\xi)=0$ for all $\xi$ not in the ball $B(\mathbf{0}, 2)$ with radius 2 and center at the origin. This proves 
the following result about the generalized Riesz potential $J_{\Omega} f$ when $f$ has vanishing moments upto some order.

Proposition 2.5 Let $m_{0} \geq 0, \alpha \in\left(-m_{0}-1, \infty\right) \backslash \mathbb{Z}$, and $\Omega \in C^{\infty}\left(\mathbb{R}^{d} \backslash\{\mathbf{0}\}\right)$ be a homogeneous function of degree $\alpha$. Then the following statements hold.

(i) If $f$ satisfies (2.14) and

$$
\sum_{|\mathbf{i}| \leq\lceil\alpha\rceil+2 m_{0}+d+2} \sup _{\xi \in \mathbb{R}^{d}}(1+\|\xi\|)^{\lceil\alpha\rceil+d}\left|\partial^{\mathbf{i}} \hat{f}(\xi)\right|<\infty,
$$

then there exists a positive constant $C$ such that

$$
\begin{aligned}
\left|J_{\Omega} f(\mathbf{x})\right| \leq C & \left(\sum_{|\mathbf{i}| \leq\lceil\alpha\rceil+2 m_{0}+d+2} \sup _{\xi \in \mathbb{R}^{d}}(1+\|\xi\|)^{\lceil\alpha\rceil+d}\left|\partial^{\mathbf{i}} \hat{f}(\xi)\right|\right) \\
& \times(1+\|\mathbf{x}\|)^{-\alpha-m_{0}-d-1} \quad \text { for all } \mathbf{x} \in \mathbb{R}^{d} .
\end{aligned}
$$

(ii) If $f$ satisfies (2.14) and

$$
\sum_{|\mathbf{i}| \leq \max (\lceil\alpha\rceil+d, 0)} \sup _{\mathbf{z} \in \mathbb{R}^{d}}\left((1+\|\mathbf{z}\|)^{\lceil\alpha\rceil+2 m_{0}+2 d+2+\epsilon}\left|\partial^{\mathbf{i}} f(\mathbf{z})\right|\right)<\infty
$$

for some $\epsilon>0$, then

$$
\begin{aligned}
\left|J_{\Omega} f(\mathbf{x})\right| \leq C & \left(\sum_{|\mathbf{i}| \leq \max (\lceil\alpha\rceil+d, 0)} \sup _{\mathbf{z} \in \mathbb{R}^{d}}(1+\|\mathbf{z}\|)^{\lceil\alpha\rceil+2 m_{0}+2 d+2+\epsilon}\left|\partial^{\mathbf{i}} f(\mathbf{z})\right|\right) \\
& \times(1+\|\mathbf{x}\|)^{-\alpha-m_{0}-d-1} \quad \text { for all } \mathbf{x} \in \mathbb{R}^{d}
\end{aligned}
$$

The conclusions in Proposition 2.5 do not apply to the generalized Riesz potential $J_{\Omega} f$ where $\Omega \in C^{\infty}\left(\mathbb{R}^{d} \backslash\{\boldsymbol{0}\}\right)$ is a homogeneous function of degree zero. In this case, applying the argument used to establish (2.16) and (2.17), we have that

$$
\left|J_{\Omega} f(\mathbf{x})\right| \leq C \sum_{|\mathbf{i}| \leq m_{0}+1} \sup _{\xi \in \mathbb{R}^{d}}\left((1+\|\xi\|)^{d+\epsilon}\left|\partial^{\mathbf{i}} \hat{f}(\xi)\right|\right)
$$


for all $\mathbf{x} \in \mathbb{R}^{d}$ with $\|\mathbf{x}\| \leq 1$, and

$$
\begin{aligned}
\left|J_{\Omega} f(\mathbf{x})\right| \leq & C(1+\|\mathbf{x}\|)^{-m_{0}-d} \\
& \times\left\{\sum_{|\mathbf{k}|=m_{0}+1,|\mathbf{j}| \leq m_{0}+d} \int_{0}^{1} \int_{\mathbb{R}^{d}}\left|\partial^{\mathbf{j}}\left(\phi(\|\mathbf{x}\| \xi) \xi^{\mathbf{k}} \Omega(\xi) \partial^{\mathbf{k}} \hat{f}(t \xi)\right)\right| d \xi d t\right\} \\
& +C(1+\|\mathbf{x}\|)^{-m_{0}-d-1} \sum_{|\mathbf{k}|=m_{0}+1,|\mathbf{j}|+|\mathbf{I}| \leq m_{0}+d+1,|\mathbf{j}| \leq m_{0}+d} \\
& \left.\int_{0}^{1} \int_{\mathbb{R}^{d}}\left|\partial^{\mathbf{j}}\left((\phi(\xi)-\phi(\|\mathbf{x}\| \xi)) \xi^{\mathbf{k}} \Omega(\xi)\right)\right| \times \mid \partial^{\mathbf{k}+\mathbf{l}} \hat{f}(t \xi)\right) \mid d \xi d t \\
& +C(1+\|\mathbf{x}\|)^{-m_{0}-d-2} \sum_{|\mathbf{k}|=m_{0}+1,|\mathbf{j}|+|\mathbf{I}| \leq m_{0}+d+2,|\mathbf{I}| \leq 1} \\
& \left.\int_{0}^{1} \int_{\mathbb{R}^{d}}\left|\partial^{\mathbf{j}}\left((\phi(\xi)-\phi(\|\mathbf{x}\| \xi)) \xi^{\mathbf{k}} \Omega(\xi)\right)\right| \times \mid \partial^{\mathbf{k}+\mathbf{l}} \hat{f}(t \xi)\right) \mid d \xi d t \\
& +C(1+\|\mathbf{x}\|)^{-m_{0}-d-1} \sum_{|\mathbf{j}| \leq m_{0}+d+1} \int_{\mathbb{R}^{d}}\left|\partial^{\mathbf{j}}((1-\phi(\xi)) \Omega(\xi) \hat{f}(\xi))\right| d \xi \\
\leq & \left.C\left(\sum_{|\mathbf{i}| \leq 2 m_{0}+d+2} \sup _{\xi \in \mathbb{R}^{d}}+\|\xi\|\right)^{d+\epsilon}\left|\partial^{\mathbf{i}} \hat{f}(\xi)\right|\right)(1+\|\mathbf{x}\|)^{-m_{0}-d-1}(2.23)
\end{aligned}
$$

for all $\mathbf{x} \in \mathbb{R}^{d}$ with $\|\mathbf{x}\| \geq 1$, where $\epsilon \in(0,1)$. Therefore

Proposition 2.6 Let $\Omega \in C^{\infty}\left(\mathbb{R}^{d} \backslash\{\mathbf{0}\}\right)$ be a homogeneous function of degree zero. Then the following statements hold.

(i) If $f$ satisfies (2.14) for some $m_{0} \geq 0$ and

$$
\sum_{|\mathbf{i}| \leq 2 m_{0}+d+2} \sup _{\xi \in \mathbb{R}^{d}}(1+\|\xi\|)^{d+\epsilon}\left|\partial^{\mathbf{i}} \hat{f}(\xi)\right|<\infty
$$

for some $\epsilon>0$, then there exists a positive constant $C$ such that

$$
\begin{aligned}
\left|J_{\Omega} f(\mathbf{x})\right| & \leq C\left(\sum_{|\mathbf{i}| \leq 2 m_{0}+d+2} \sup _{\xi \in \mathbb{R}^{d}}(1+\|\xi\|)^{d+\epsilon}\left|\partial^{\mathbf{i}} \hat{f}(\xi)\right|\right) \\
& \times(1+\|\mathbf{x}\|)^{-m_{0}-d-1} \quad \text { for all } \mathbf{x} \in \mathbb{R}^{d} .
\end{aligned}
$$

(ii) If $f$ satisfies (2.14) for some $m_{0} \geq 0$ and

$$
\sum_{|\mathbf{i}| \leq d+1} \sup _{\mathbf{z} \in \mathbb{R}^{d}}\left((1+\|\mathbf{z}\|)^{2 m_{0}+2 d+2+\epsilon}\left|\partial^{\mathbf{i}} f(\mathbf{z})\right|\right)<\infty
$$

for some $\epsilon>0$, then

$$
\begin{aligned}
\left|J_{\Omega} f(\mathbf{x})\right| \leq C & \left(\sum_{|\mathbf{i}| \leq d+1} \sup _{\mathbf{z} \in \mathbb{R}^{d}}\left((1+\|\mathbf{z}\|)^{2 m_{0}+2 d+2+\epsilon}\left|\partial^{\mathbf{i}} f(\mathbf{z})\right|\right)\right. \\
& \times(1+\|\mathbf{x}\|)^{-m_{0}-d-1} \quad \text { for all } \mathbf{x} \in \mathbb{R}^{d} .
\end{aligned}
$$


2.2 Translation-invariance and dilation-invariance

In this subsection, we show that the generalized Riesz potential $J_{\Omega}$ from $\mathcal{S}$ to $\mathcal{S}^{\prime}$ is dilation-invariant and translation-invariant, and that its restriction on the closed subspace $\mathcal{S}_{\infty}$ of $\mathcal{S}$ is the same as the linear operator $i_{\Omega}$ on $\mathcal{S}_{\infty}$.

Theorem 2.7 Let $\gamma \in \mathbb{R}$ with $\gamma-d \notin \mathbb{Z}_{+}, \Omega \in C^{\infty}\left(\mathbb{R}^{d} \backslash\{\mathbf{0}\}\right)$ be a homogeneous function of degree $-\gamma$, and let $J_{\Omega}$ be defined by (2.1). Then

(i) $J_{\Omega}$ is dilation-invariant;

(ii) $J_{\Omega}$ is translation-invariant; and

(iii) $\widehat{J_{\Omega} f}(\xi)=\Omega(\xi) \hat{f}(\xi)$ for any function $f \in \mathcal{S}_{\infty}$.

Proof (i) For any $f \in \mathcal{S}$ and any $t>0$,

$$
\begin{aligned}
J_{\Omega}\left(\delta_{t} f\right)(\mathbf{x})= & \frac{(2 \pi t)^{-d} \Gamma(d-\gamma)}{\Gamma\left(d+k_{0}-\gamma\right)} \int_{S^{d-1}} \int_{0}^{\infty} \Omega\left(\xi^{\prime}\right) r^{k_{0}-\gamma+d-1} \\
& \times\left(-\frac{d}{d r}\right)^{k_{0}}\left(e^{i r\left(\mathbf{x}, \xi^{\prime}\right\rangle} \hat{f}\left(r \xi^{\prime} / t\right)\right) d r d \sigma\left(\xi^{\prime}\right)=t^{-\gamma} \delta_{t}\left(J_{\Omega} f\right)(\mathbf{x}),
\end{aligned}
$$

where the first equality follows from $\widehat{\delta_{t} f}(\xi)=t^{-d} \hat{f}(\xi / t)$ and the second equality is obtained by change of variables. This leads to the dilation-invariance of the generalized Riesz potential $J_{\Omega}$.

(ii) For any $f \in \mathcal{S}$ and a vector $\mathbf{x}_{0} \in \mathbb{R}^{d}$, we obtain from (2.1) that

$$
\begin{aligned}
J_{\Omega}\left(\tau_{\mathbf{x}_{0}} f\right)(\mathbf{x})= & \frac{(2 \pi)^{-d} \Gamma(d-\gamma)}{\Gamma\left(d+k_{0}-\gamma\right)} \int_{S^{d-1}} \int_{0}^{\infty} r^{k_{0}-\gamma+d-1} \Omega\left(\xi^{\prime}\right) \\
& \times\left(-\frac{d}{d r}\right)^{k_{0}}\left(e^{i r\left\langle\mathbf{x}-\mathbf{x}_{0}, \xi^{\prime}\right\rangle} \hat{f}\left(r \xi^{\prime}\right)\right) d r d \sigma\left(\xi^{\prime}\right)=J_{\Omega} f\left(\mathbf{x}-\mathbf{x}_{0}\right),
\end{aligned}
$$

where $k_{0}$ is a nonnegative integer larger than $\gamma-d$. This shows that the generalized Riesz potential $J_{\Omega}$ is translation-invariant.

(iii) The third conclusion follows by taking Fourier transform of the equation (2.5) on both sides.

\subsection{Composition and left-inverse}

In this subsection, we consider the composition and left-inverse properties of generalized Riesz potentials.

Theorem 2.8 Let $\gamma_{1}$ and $\gamma_{2} \in \mathbb{R}$ satisfy $\gamma_{2}<d, \gamma_{1}+\gamma_{2}<d$ and $\gamma_{1}-d \notin \mathbb{Z}_{+}$, and let $\Omega_{1}, \Omega_{2} \in C^{\infty}\left(\mathbb{R}^{d} \backslash\{\mathbf{0}\}\right)$ be homogeneous functions of degree $-\gamma_{1}$ and $-\gamma_{2}$ respectively. Then

$$
J_{\Omega_{1}}\left(J_{\Omega_{2}} f\right)=J_{\Omega_{1} \Omega_{2}} f \text { for all } f \in \mathcal{S} \text {. }
$$


As a consequence of Theorem 2.8, we have the following result about leftinvertibility of the generalized Riesz potential $J_{\Omega}$.

Corollary 2.9 Let $\gamma \in(-d, \infty)$ with $\gamma-d \notin \mathbb{Z}_{+}$and $\Omega \in C^{\infty}\left(\mathbb{R}^{d} \backslash\{\mathbf{0}\}\right)$ be homogeneous of degree $-\gamma$ with $\Omega(\xi) \neq 0$ for all $\xi \in S^{d-1}$. Then $J_{\Omega} J_{\Omega^{-1}}$ is an identity operator on $\mathcal{S}$. If we further assume that $\gamma \in(-d, d)$, then both $J_{\Omega^{-1}} J_{\Omega}$ and $J_{\Omega} J_{\Omega^{-1}}$ are identity operators on $\mathcal{S}$.

Taking $\Omega(\xi)=\|\xi\|^{-\gamma}$ in the above corollary yields that the linear operator $I_{\gamma}$ in (1.10) is a left-inverse of the fractional Laplacian $(-\triangle)^{\gamma / 2}$.

Corollary 2.10 Let $\gamma$ be a positive number with $\gamma-d \notin \mathbb{Z}_{+}$. Then $I_{\gamma}$ is a leftinverse of the fractional Laplacian $(-\triangle)^{\gamma / 2}$.

Proof of Theorem 2.8 Let $k_{0}$ be the smallest nonnegative integer such that $k_{0}-\gamma_{1}+d>0$, and set $\Omega(\xi)=\Omega_{1}(\xi) \Omega_{2}(\xi)$. If $k_{0}=0$, then the conclusion (2.24) follows from (2.2). Now we assume that $k_{0} \geq 1$. Then

$$
\begin{aligned}
J_{\Omega_{1}}\left(J_{\Omega_{2}} f\right)(\mathbf{x})= & \frac{(2 \pi)^{d} \Gamma\left(d-\gamma_{1}\right)}{\Gamma\left(d+k_{0}-\gamma_{1}\right)} \lim _{\epsilon \rightarrow 0} \int_{S^{d-1}} \int_{\epsilon}^{\infty} \Omega\left(\xi^{\prime}\right) r^{k_{0}+d-\gamma_{1}-1} \\
& \times\left\{r\left(-\frac{d}{d r}\right)^{k_{0}}\left(e^{i r\left\langle\mathbf{x}, \xi^{\prime}\right\rangle} \hat{f}\left(r \xi^{\prime}\right) r^{-\gamma_{2}-1}\right)\right. \\
& \left.-k_{0}\left(-\frac{d}{d r}\right)^{k_{0}-1}\left(e^{i r\left\langle\mathbf{x}, \xi^{\prime}\right\rangle} \hat{f}\left(r \xi^{\prime}\right) r^{-\gamma_{2}-1}\right)\right\} d r d \sigma\left(\xi^{\prime}\right) \\
= & \frac{(2 \pi)^{d} \Gamma\left(d+1-\gamma_{1}\right)}{\Gamma\left(d+k_{0}-\gamma_{1}\right)} \lim _{\epsilon \rightarrow 0} \int_{S^{d-1}} \int_{\epsilon}^{\infty} \Omega\left(\xi^{\prime}\right) r^{k_{0}+d-\gamma_{1}-1} \\
& \times\left(-\frac{d}{d r}\right)^{k_{0}-1}\left(e^{i r\left\langle\mathbf{x}, \xi^{\prime}\right\rangle} \hat{f}\left(r \xi^{\prime}\right) r^{-\gamma_{2}-1}\right) d r d \sigma\left(\xi^{\prime}\right) \\
= & \cdots \\
= & \frac{(2 \pi)^{-d} \Gamma\left(d+k_{0}-\gamma_{1}\right)}{\Gamma\left(d+k_{0}-\gamma_{1}\right)} \lim _{\epsilon \rightarrow 0} \int_{S^{d-1}} \int_{\epsilon}^{\infty} \Omega\left(\xi^{\prime}\right) r^{k_{0}+d-\gamma_{1}-1} \\
& \times\left(e^{i r\left\langle\mathbf{x}, \xi^{\prime}\right\rangle} \hat{f}\left(r \xi^{\prime}\right) r^{-\gamma_{2}-k_{0}}\right) d r d \sigma\left(\xi^{\prime}\right) \\
= & J_{\Omega_{1} \Omega 2} f(\mathbf{x}) \text { for all } \mathbf{x} \in \mathbb{R}^{d},
\end{aligned}
$$

where the second equality is obtained by applying the integration-byparts technique and using the fact that $\left.\epsilon^{k_{0}+d-\gamma_{1}}\left(\frac{d}{d r}\right)^{k_{0}-1}\left(e^{\left.i r / \mathbf{x}, \xi^{\prime}\right\rangle} \hat{f}\left(r \xi^{\prime}\right) r^{-\gamma_{2}-1}\right)\right|_{r=\epsilon}$ 
converges to zero uniformly on $\xi \in S^{d-1}$ under the assumption that $\gamma_{1}+\gamma_{2}<$ $d$. The conclusion (2.24) then follows.

\subsection{Translation-invariant and dilation-invariant extensions of the linear operator $i_{\Omega}$}

In this subsection, we show that the generalized Riesz potential $J_{\Omega}$ in (2.1) is the only continuous linear operator from $\mathcal{S}$ to $\mathcal{S}^{\prime}$ that is translation-invariant and dilation-invariant, and that is an extension of the linear operator $i_{\Omega}$ in (2.4) from the closed subspace $\mathcal{S}_{\infty}$ to the whole space $\mathcal{S}$.

Theorem 2.11 Let $\gamma$ be a positive number with $\gamma-d \notin \mathbb{Z}_{+}, \Omega \in C^{\infty}\left(\mathbb{R}^{d} \backslash\{\mathbf{0}\}\right)$ be a nonzero homogeneous function of degree $-\gamma$, and let $J_{\Omega}$ be defined by (2.1). Then I is a continuous linear operator from $\mathcal{S}$ to $\mathcal{S}^{\prime}$ such that I is dilationinvariant and translation-invariant, and that the restriction of I on $\mathcal{S}_{\infty}$ is the same as the linear operator $i_{\Omega}$ in (2.4) if and only if $I=J_{\Omega}$.

To prove Theorem 2.11, we need two technical lemmas about extensions of the linear operator $i_{\Omega}$ on $\mathcal{S}_{\infty}$.

Lemma 2.12 Let $\gamma$ be a positive number with $\gamma-d \notin \mathbb{Z}_{+}, \Omega \in C^{\infty}\left(\mathbb{R}^{d} \backslash\{\mathbf{0}\}\right)$ be a homogeneous function of degree $-\gamma$, and let $J_{\Omega}$ be defined by (2.1). Then a continuous linear operator I from $\mathcal{S}$ to $\mathcal{S}^{\prime}$ is an extension of the linear operator $i_{\Omega}$ on $\mathcal{S}_{\infty}$ if and only if

$$
I f=J_{\Omega} f+\sum_{|\mathbf{i}| \leq N} \frac{\partial^{\mathbf{i}} \hat{f}(\mathbf{0})}{\mathbf{i} !} H_{\mathbf{i}}
$$

for some integer $N$ and tempered distributions $H_{\mathbf{i}}, \mathbf{i} \in \mathbb{Z}_{+}^{d}$ with $|\mathbf{i}| \leq N$.

Proof The sufficiency follows from Theorem 2.7 and the assumption that $H_{\mathbf{i}},|\mathbf{i}| \leq N$, in (2.25) are tempered distributions. Now the necessity. By Corollary 2.3 and Theorem $2.7, I-J_{\Omega}$ is a continuous linear operator from $\mathcal{S}$ to $\mathcal{S}^{\prime}$ that satisfies that $\left(I-J_{\Omega}\right) f=0$ for all $f \in \mathcal{S}_{\infty}$. This implies that the inverse Fourier transform of the tempered distribution $\left(I-J_{\Omega}\right)^{*} g$ is supported on the origin for any Schwartz function $g$. Hence there exist an integer $N$ and tempered distribution $H_{\mathbf{i}},|\mathbf{i}| \leq N$, such that $\mathcal{F}^{-1}\left(\left(I-J_{\Omega}\right)^{*} g\right)=$ $\sum_{|\mathbf{i}| \leq N}\left\langle g, H_{\mathbf{i}}\right\rangle \delta^{(\mathbf{i})} / \mathbf{i}$ !, where the tempered distributions $\delta^{(\mathbf{i})}, \mathbf{i} \in \mathbb{Z}_{+}^{d}$, are defined by $\left\langle\delta^{(\mathbf{i})}, f\right\rangle=\partial^{\mathbf{i}} f(\mathbf{0})$ [7, Theorem 2.3.4]. Then $\left\langle\left(I-J_{\Omega}\right) f, g\right\rangle=\left\langle\hat{f}, \mathcal{F}^{-1}(I-\right.$ $\left.\left.J_{\Omega}\right)^{*} g\right\rangle=\sum_{|\mathbf{i}| \leq N}\left\langle H_{\mathbf{i}}, g\right\rangle \partial^{\mathbf{i}} \hat{f}(\mathbf{0}) / \mathbf{i}$ ! for all Schwartz functions $f$ and $g$, and hence (2.25) is established. 
Lemma 2.13 Let $\gamma$ be a positive number with $\gamma-d \notin \mathbb{Z}_{+}$, and consider the continuous linear operator $K$ from $\mathcal{S}$ to $\mathcal{S}^{\prime}$ :

$$
K f=\sum_{|\mathbf{i}| \leq N} \frac{\partial^{\mathbf{i}} \hat{f}(\mathbf{0})}{\mathbf{i} !} H_{\mathbf{i}}, \quad f \in \mathcal{S}
$$

where $N \in \mathbb{Z}_{+}$and $H_{\mathbf{i}},|\mathbf{i}| \leq N$, are tempered distributions, Then the following statements hold.

(i) The equation

$$
K\left(\delta_{t} f\right)=t^{-\gamma} \delta_{t}(K f)
$$

holds for any $f \in \mathcal{S}$ and $t>0$ if and only if for every $\mathbf{i} \in \mathbb{Z}_{+}^{d}$ with $|\mathbf{i}| \leq N$, $H_{\mathbf{i}}$ is homogeneous of degree $\gamma-d-|\mathbf{i}|$.

(ii) The linear operator $K$ is translation-invariant if and only if there exists a polynomial $P$ of degree at most $N$ such that $H_{\mathbf{i}}=(-i \partial)^{\mathbf{i}} P$ for all $\mathbf{i} \in \mathbb{Z}_{+}^{d}$ with $|\mathbf{i}| \leq N$.

(iii) The linear operator $K$ is translation-invariant and satisfies (2.27) if and only if $H_{\mathbf{i}}=0$ for all $\mathbf{i} \in \mathbb{Z}_{+}^{d}$ with $|\mathbf{i}| \leq N$.

Proof (i) The sufficiency follows from the homogeneous assumption on $H_{\mathbf{i}},|\mathbf{i}| \leq N$, and the observation that

$$
\partial^{\mathbf{i}} \widehat{\delta_{t} f}(\mathbf{0})=t^{-d-|\mathbf{i}|} \partial^{\mathbf{i}} \hat{f}(\mathbf{0}) \quad \text { for all } f \in \mathcal{S} \text { and } \mathbf{i} \in \mathbb{Z}_{+}^{d} .
$$

Now the necessity. Let $\phi$ be a $C^{\infty}$ function such that $\phi(\xi)=1$ for all $\xi \in B(\mathbf{0}, 1)$ and $\phi(\xi)=0$ for all $\xi \notin B(\mathbf{0}, 2)$, where $B(\mathbf{x}, r)$ is the ball with center $\mathbf{x} \in \mathbb{R}^{d}$ and radius $r>0$. Define $\psi_{\mathbf{i}} \in \mathcal{S}, \mathbf{i} \in \mathbb{Z}_{+}^{d}$, with the help of the Fourier transform by

$$
\widehat{\psi}_{\mathbf{i}}(\xi)=\frac{\xi^{\mathbf{i}}}{\mathbf{i} !} \phi(\xi)
$$

One may verify that

$$
\partial^{\mathbf{i}^{\prime}} \widehat{\psi_{\mathbf{i}}}(\mathbf{0})=\left\{\begin{array}{l}
1 \text { if } \mathbf{i}^{\prime}=\mathbf{i} \\
0 \text { if } \mathbf{i}^{\prime} \neq \mathbf{i}
\end{array}\right.
$$

For any $\mathbf{i} \in \mathbb{Z}_{+}^{d}$ with $|\mathbf{i}| \leq N$, the homogeneous property of the tempered distribution $H_{\mathbf{i}}$ follows by replacing $f$ in (2.27) by $\psi_{\mathbf{i}}$ and using (2.30). 
(ii) $(\Longleftarrow)$ Given $f \in \mathcal{S}$ and $\mathbf{x}_{0} \in \mathbb{R}^{d}$,

$$
\begin{aligned}
K\left(\tau_{\mathbf{x}_{0}} f\right)(\mathbf{x}) & =\sum_{|\mathbf{i}| \leq N} \sum_{\mathbf{j}+\mathbf{k}=\mathbf{i}} \frac{\left(-i \mathbf{x}_{0}\right)^{\mathbf{k}}}{\mathbf{k} !} \frac{\partial^{\mathbf{j}} \hat{f}(\mathbf{0})}{\mathbf{j} !}(-i \partial)^{\mathbf{i}} P(\mathbf{x}) \\
& =\sum_{|\mathbf{j}| \leq N} \frac{(-i)^{\mathbf{j}} \partial^{\mathbf{j}} \hat{f}(\mathbf{0})}{\mathbf{j} !}\left(\sum_{|\mathbf{k}| \leq N-|\mathbf{j}|} \frac{\partial^{\mathbf{j}+\mathbf{k}} P(\mathbf{x})}{\mathbf{k} !}\left(-\mathbf{x}_{0}\right)^{\mathbf{k}}\right) \\
& =\sum_{|\mathbf{j}| \leq N} \frac{(-i)^{\mathbf{j}} \partial^{\mathbf{j}} \hat{f}(\mathbf{0})}{\mathbf{j} !} \partial^{\mathbf{j}} P\left(\mathbf{x}-\mathbf{x}_{0}\right)=K f\left(\mathbf{x}-\mathbf{x}_{0}\right),
\end{aligned}
$$

where the first equality follows from

$$
\partial^{\mathbf{i}} \widehat{\tau_{\mathbf{x}_{0}} f}(\mathbf{0})=\sum_{\mathbf{j} \leq \mathbf{i}}\left(\begin{array}{l}
\mathbf{i} \\
\mathbf{j}
\end{array}\right)\left(-i \mathbf{x}_{0}\right)^{\mathbf{i}-\mathbf{j}} \partial^{\mathbf{j}} \hat{f}(\mathbf{0}),
$$

and the third equality is deducted from the Taylor expression of the polynomial $\partial^{\mathbf{j}} P$ of degree at most $N-|\mathbf{j}|$.

$(\Longrightarrow)$ By (2.32) and the translation-invariance of the linear operator $K$,

$$
\sum_{|\mathbf{i}| \leq N} \sum_{\mathbf{j}+\mathbf{k}=\mathbf{i}} \frac{\left(-i \mathbf{x}_{0}\right)^{\mathbf{k}}}{\mathbf{k} !} \frac{\partial^{\mathbf{j}} \hat{f}(\mathbf{0})}{\mathbf{j} !} H_{\mathbf{i}}=\sum_{|\mathbf{i}| \leq N} \frac{\partial^{\mathbf{j}} \hat{f}(\mathbf{0})}{\mathbf{j} !} \tau_{\mathbf{x}_{0}} H_{\mathbf{j}}
$$

holds for any Schwartz function $f$ and $\mathbf{x}_{0} \in \mathbb{R}^{d}$. Replacing $f$ in the above equation by the function $\psi_{0}$ in (2.29) and then using (2.30), we get

$$
\tau_{\mathbf{x}_{0}} H_{\mathbf{0}}=\sum_{|\mathbf{i}| \leq N} \frac{\left(-i \mathbf{x}_{0}\right)^{\mathbf{i}}}{\mathbf{i} !} H_{\mathbf{i}}
$$

This implies that $\left\langle H_{\mathbf{0}}, g\left(\cdot+\mathbf{x}_{0}\right)\right\rangle=\sum_{|\mathbf{i}| \leq N} \frac{\left(-i \mathbf{x}_{0}\right)^{\mathbf{i}}}{\mathbf{i} !}\left\langle H_{\mathbf{i}}, g\right\rangle$ for any Schwartz function $g$. By taking partial derivatives $\partial^{\mathbf{k}},|\mathbf{k}|=N+1$, with respect to $\mathbf{x}_{0}$ of both sides of the above equation, using the fact that $\partial^{\mathbf{k}} \mathbf{x}^{\mathbf{i}}=0$ for all $\mathbf{k} \in \mathbb{Z}_{+}$with $|\mathbf{k}|=N+1$, and then letting $\mathbf{x}_{0}=\mathbf{0}$, we obtain that $\left\langle H_{\mathbf{0}}, \partial^{\mathbf{k}} g\right\rangle=0$ holds for any $g \in \mathcal{S}$ and $\mathbf{k} \in \mathbb{Z}_{+}$with $|\mathbf{k}|=N+1$. Hence $H_{\mathbf{0}}=P$ for some polynomial $P$ of degree at most $N$. The desired conclusion about $H_{\mathbf{i}},|\mathbf{i}| \leq N$, then follows from (2.34) and $\tau_{\mathbf{x}_{0}} H_{\mathbf{0}}(\mathbf{x})=\sum_{|\mathbf{i}| \leq N} \frac{\left(-\mathbf{x}_{0}\right)^{\mathbf{i}}}{\mathbf{i} !} \partial^{\mathbf{i}} P(\mathbf{x})$ by the Taylor expansion of the polynomial $P$.

(iii) Clearly if $H_{\mathbf{i}}=0$ for all $|\mathbf{i}| \leq N$, then $K f=0$ for all $f \in \mathcal{S}$ and hence $K$ is translation-invariant and satisfies (2.27). Conversely, if $K$ is translationinvariant and satisfies (2.27), it follow from the conclusions (i) and (ii) that for every $\mathbf{i} \in \mathbb{Z}_{+}^{d}$ with $|\mathbf{i}| \leq N, H_{\mathbf{i}}$ is homogeneous of degree $\gamma-d-|\mathbf{i}| \notin \mathbb{Z}$ and also a polynomial of degree at most $N-|\mathbf{i}|$. Then $H_{\mathbf{i}}=0$ for all $\mathbf{i} \in \mathbb{Z}_{+}^{d}$ with $|\mathbf{i}| \leq N$ because the homogeneous degree of any nonzero polynomial is a nonnegative integer if it is homogeneous. 
We now have all of ingredients to prove Theorem 2.11.

Proof of Theorem 2.11 The sufficiency follows from Corollary 2.3 and Theorem 2.7. Now the necessity. By Lemma 2.12, there exist an integer $N$ and tempered distributions $H_{\mathbf{i}},|\mathbf{i}| \leq N$, such that (2.25) holds. Define $K f=$ $\sum_{|\mathbf{i}| \leq N} \frac{\partial^{\mathbf{i}} \hat{f}(\mathbf{0})}{\mathbf{i} !} H_{\mathbf{i}}$ for any $f \in \mathcal{S}$. Then $K f$ is a continuous linear operator from $\mathcal{S}$ to $\mathcal{S}^{\prime}$ and

$$
\text { If }=J_{\Omega} f+K f, \quad f \in \mathcal{S} .
$$

Moreover the linear operator $K$ satisfies (2.27) and is translation-invariant by (2.35), Theorem 2.7 and the assumption on $I$. Then $K f=0$ for all $f \in \mathcal{S}$ by Lemma 2.13. This together with (2.35) proves the desired conclusion that $I=J_{\Omega}$.

2.5 Translation-invariant extensions of the linear operator $i_{\Omega}$ with additional localization in the Fourier domain

Given a nonzero homogeneous function $\Omega \in C^{\infty}\left(\mathbb{R}^{d} \backslash\{\boldsymbol{0}\}\right)$ of degree $-\gamma$, we recall from (2.2) and Theorem 2.7 that $J_{\Omega}$ is translation-invariant and the Fourier transform of $J_{\Omega} f$ belongs to $K_{1}$ when $\gamma \in(0, d)$, where

$$
K_{1}=\left\{h: \int_{\mathbb{R}^{d}}|h(\xi)|(1+\|\xi\|)^{-N} d \xi<\infty \text { for some } N \geq 1\right\}
$$

In fact, the generalized Riesz potential $J_{\Omega}$ is the only extension of the linear operator $i_{\Omega}$ on $\mathcal{S}_{\infty}$ to the whole space $\mathcal{S}$ with the above two properties.

Theorem 2.14 Let $\gamma>0$ with $\gamma-d \notin \mathbb{Z}_{+}, \Omega \in C^{\infty}\left(\mathbb{R}^{d} \backslash\{\mathbf{0}\}\right)$ be a nonzero homogeneous function of degree $-\gamma$, and the continuous linear operator I from $\mathcal{S}$ to $\mathcal{S}^{\prime}$ be an extension of the linear operator $i_{\Omega}$ on $\mathcal{S}_{\infty}$ such that the Fourier transform of If belongs to $K_{1}$ for all $f \in \mathcal{S}$. Then I is translation-invariant if and only if $I=J_{\Omega}$ and $\gamma \in(0, d)$.

Proof The sufficiency follows from (2.2) and Theorem 2.7. Now we prove the necessity. By the assumption on the linear operator $I$, applying an argument similar to the proof of Lemma 2.12, we can find a family of functions $g_{\mathbf{i}} \in$ $K_{1},|\mathbf{i}| \leq N$, such that

$$
\widehat{I f}(\xi)=\left(\hat{f}(\xi)-\sum_{|\mathbf{i}| \leq \gamma-d} \frac{\partial^{\mathbf{i}} \hat{f}(\mathbf{0})}{\mathbf{i} !} \xi^{\mathbf{i}}\right) \Omega(\xi)+\sum_{|\mathbf{i}| \leq N} \frac{\partial^{\mathbf{i}} \hat{f}(\mathbf{0})}{\mathbf{i} !} g_{\mathbf{i}}(\xi)
$$


for any Schwartz function $f$. This together with (2.32) and the translationinvariance of the linear operator $I$ implies that

$$
\begin{aligned}
& -\sum_{|\mathbf{i}| \leq \gamma-d} \sum_{\mathbf{j}+\mathbf{k}=\mathbf{i}} \frac{\partial^{\mathbf{j}} \hat{f}(\mathbf{0})}{\mathbf{k} ! \mathbf{j} !}\left(-i \mathbf{x}_{0}\right)^{\mathbf{k}} \xi^{\mathbf{i}} \Omega(\xi)+\sum_{|\mathbf{i}| \leq N} \sum_{\mathbf{j}+\mathbf{k}=\mathbf{i}} \frac{\partial^{\mathbf{j}} \hat{f}(\mathbf{0})}{\mathbf{k} ! \mathbf{j} !}\left(-i \mathbf{x}_{0}\right)^{\mathbf{k}} g_{\mathbf{i}}(\xi) \\
= & e^{i \mathbf{x}_{0} \xi}\left(-\sum_{|\mathbf{i}| \leq \gamma-d} \frac{\partial^{\mathbf{i}} \hat{f}(\mathbf{0})}{\mathbf{i} !} \xi^{\mathbf{i}} \Omega(\xi)+\sum_{|\mathbf{i}| \leq N} \frac{\partial^{\mathbf{i}} \hat{f}(\mathbf{0})}{\mathbf{i} !} g_{\mathbf{i}}(\xi)\right) .
\end{aligned}
$$

As $\mathbf{x}_{0} \in \mathbb{R}^{d}$ in (2.38) is chosen arbitrarily, we conclude that

$$
-\sum_{|\mathbf{i}| \leq \gamma-d} \frac{\partial^{\mathbf{i}} \hat{f}(\mathbf{0})}{\mathbf{i} !} \xi^{\mathbf{i}} \Omega(\xi)+\sum_{|\mathbf{i}| \leq N} \frac{\partial^{\mathbf{i}} \hat{f}(\mathbf{0})}{\mathbf{i} !} g_{\mathbf{i}}(\xi)=0 \quad \text { for all } f \in \mathcal{S} .
$$

Substituting the above equation into (2.37), we then obtain $\widehat{I f}(\xi)=\hat{f}(\xi) \Omega(\xi)$ for all $f \in \mathcal{S}$. This, together with the observation that $\hat{f} \Omega \in K_{1}$ for all $f \in \mathcal{S}$ if and only if $\gamma<d$, leads to the desired conclusion that $I=J_{\Omega}$ and $\gamma \in(0, d)$.

\subsection{Non-integrability in the spatial domain}

Let $\gamma>0$ with $\gamma-d \notin \mathbb{Z}_{+}$and $\Omega \in C^{\infty}\left(\mathbb{R}^{d} \backslash\{\boldsymbol{0}\}\right)$ be a nonzero homogeneous function of degree $-\gamma$. For any Schwartz function $f$, there exists a positive constant $C$ by Theorem 2.1 such that $\left|J_{\Omega} f(\mathbf{x})\right| \leq C(1+\|\mathbf{x}\|)^{\gamma-d}$ for all $\mathbf{x} \in$ $\mathbb{R}^{d}$. Hence $J_{\Omega} f \in L^{p}, 1 \leq p \leq \infty$, when $\gamma<d(1-1 / p)$. In this subsection, we show that the above $p$-integrability property for the generalized Riesz potential $J_{\Omega}$ is no longer true when $\gamma \geq d(1-1 / p)$.

Theorem 2.15 Let $1 \leq p \leq \infty, 0<\gamma \in[d(1-1 / p), \infty) \backslash \mathbb{Z}$ and $\Omega \in C^{\infty}\left(\mathbb{R}^{d} \backslash\{\mathbf{0}\}\right)$ be a nonzero homogeneous function of degree $-\gamma$. Then there exists a Schwartz function $f$ such that $J_{\Omega} f \notin L^{p}$.

Letting $\Omega(\xi)=\|\xi\|^{-\gamma}$ in Theorem 2.15 leads to the conclusion mentioned in the abstract:

Corollary 2.16 Let $1 \leq p \leq \infty$ and $d(1-1 / p) \leq \gamma \notin \mathbb{Z}_{+}$. Then $I_{\gamma} f$ is not $p$ integrable for some function $f \in \mathcal{S}$.

Proof of Theorem 2.15 Let the Schwartz functions $\phi$ and $\psi_{\mathbf{i}}, \mathbf{i} \in \mathbb{Z}_{+}^{d}$, be as in the proof of Lemma 2.13. We examine three cases to prove the theorem. 
Case $I \quad d(1-1 / p) \leq \gamma<\min (d, d(1-1 / p)+1)$ In this case, $1 \leq p<\infty$ and

$$
J_{\Omega} \psi_{\mathbf{0}}(\mathbf{x})=\int_{\mathbb{R}^{d}} K(\mathbf{x}-\mathbf{y}) \psi_{\mathbf{0}}(\mathbf{y}) d \mathbf{y}
$$

by (2.2), where $K$ is the inverse Fourier transform of $\Omega$. By [7, Theorems 7.1.16 and 7.1.18], $K \in C^{\infty}\left(\mathbb{R}^{d} \backslash\{0\}\right)$ is a homogeneous function of order $\gamma-d \in$ $(-d, 0)$, which implies that

$$
\left|\partial^{\mathbf{i}} K(\mathbf{x})\right| \leq C\|\mathbf{x}\|^{\gamma-d-|\mathbf{i}|} \quad \text { for all } \mathbf{i} \in \mathbb{Z}_{+}^{d} \text { with }|\mathbf{i}| \leq 1 .
$$

Using (2.38) and (2.39), and noting that $\psi_{\mathbf{0}} \in \mathcal{S}$ satisfies $\int_{\mathbb{R}^{d}} \psi_{\mathbf{0}}(\mathbf{y}) d \mathbf{y}=1$, we obtain that for all $\mathbf{x} \in \mathbb{R}^{d}$ with $\|\mathbf{x}\| \geq 1$,

$$
\begin{aligned}
\left|J_{\Omega} \psi_{\mathbf{0}}(\mathbf{x})-K(\mathbf{x})\right| \leq & \int_{\|\mathbf{y}\| \leq\|\mathbf{x}\| / 2}\left|K(\mathbf{x}-\mathbf{y})-K(\mathbf{x}) \| \psi_{\mathbf{0}}(\mathbf{y})\right| d \mathbf{y} \\
& +\left(\int_{\|\mathbf{x}\| / 2 \leq\|\mathbf{y}\| \leq 2\|\mathbf{x}\|}+\int_{2\|\mathbf{x}\| \leq\|\mathbf{y}\|}\right)\left|K(\mathbf{x}-\mathbf{y}) \| \psi_{\mathbf{0}}(\mathbf{y})\right| d \mathbf{y} \\
& +|K(\mathbf{x})| \int_{\|\mathbf{y}\| \geq\|\mathbf{x}\| / 2}\left|\psi_{\mathbf{0}}(\mathbf{y})\right| d \mathbf{y} \\
\leq & C(1+\|\mathbf{x}\|)^{\gamma-d-1} .
\end{aligned}
$$

We notice that $\int_{\|\mathbf{x}\| \geq 1}(1+\|\mathbf{x}\|)^{(\gamma-d-1) p} d \mathbf{x}<\infty$ and $\int_{\|\mathbf{x}\| \geq 1}|K(\mathbf{x})|^{p} d \mathbf{x}=\infty$ because $K$ is a nonzero homogenous function of degree $\gamma-d$ and $d-p<$ $(d-\gamma) p \leq d$. The above two observations together with the estimate in (2.40) prove that $J_{\Omega} \psi_{\mathbf{0}} \notin L^{p}$, the desired conclusion with $f=\psi_{\mathbf{0}}$.

Case II $d<\gamma<d(1-1 / p)+1$. In this case, $d<p \leq \infty$ and

$$
J_{\Omega} \psi_{\mathbf{0}}(\mathbf{x})=\frac{1}{d-\gamma} \sum_{|\mathbf{j}|=1} J_{\Omega_{\mathbf{j}}}\left(\varphi_{\mathbf{j}}\right)(\mathbf{x})+\frac{1}{d-\gamma} \sum_{|\mathbf{i}|=1}(-\mathbf{x})^{\mathbf{i}} J_{\Omega_{\mathbf{i}}} \psi_{\mathbf{0}}(\mathbf{x})
$$

by taking $k_{0}=1$ in (2.8), where $\Omega_{\mathbf{i}}(\xi)=(i \xi)^{\mathbf{i}} \Omega(\xi)$ and $\varphi_{\mathbf{i}}(\mathbf{x})=\mathbf{x}^{\mathbf{i}} \psi_{\mathbf{0}}(\mathbf{x})$. Let $K_{\mathbf{i}}$ be the inverse Fourier transform of the function $\Omega_{\mathbf{i}}$, $|\mathbf{i}|=1$. Noticing that $\Omega_{\mathbf{i}}$ is homogeneous of degree $-\gamma+1$ and that $\int_{\mathbb{R}^{d}} \varphi_{\mathbf{i}}(\mathbf{x}) d \mathbf{x}=0$, we then apply similar argument to the one used in establishing (2.40) and obtain

$$
\left|J_{\Omega_{\mathbf{i}}}\left(\varphi_{\mathbf{i}}\right)(\mathbf{x})\right|+\left|J_{\Omega_{\mathbf{i}}} \psi_{\mathbf{0}}(\mathbf{x})-K_{\mathbf{i}}(\mathbf{x})\right| \leq C\|\mathbf{x}\|^{\gamma-d-2} \quad \text { if }\|\mathbf{x}\| \geq 1
$$

Hence

$$
\int_{\|\mathbf{x}\| \geq 1}\left|J_{\Omega} \psi_{\mathbf{0}}(\mathbf{x})-\frac{1}{d-\gamma} \sum_{|\mathbf{i}|=1}(-\mathbf{x})^{\mathbf{i}} K_{\mathbf{i}}(\mathbf{x})\right|^{p} d \mathbf{x} \leq C \int_{\|\mathbf{x}\| \geq 1}\|\mathbf{x}\|^{(\gamma-d-1) p} d \mathbf{x}<\infty
$$


if $d<p<\infty$ and

$$
\sup _{\|\mathbf{x}\| \geq 1}\left|J_{\Omega} \psi_{\mathbf{0}}(\mathbf{x})-\frac{1}{d-\gamma} \sum_{|\mathbf{i}|=1}(-i \mathbf{x})^{\mathbf{i}} K_{\mathbf{i}}(\mathbf{x})\right| \leq C \sup _{\|\mathbf{x}\| \geq 1}\|\mathbf{x}\|^{\gamma-d-1}<\infty
$$

if $p=\infty$. Set $K(\mathbf{x}):=\sum_{|\mathbf{i}|=1}(-\mathbf{x})^{\mathbf{i}} K_{\mathbf{i}}(\mathbf{x})$. Then $K$ is homogeneous of degree $\gamma-$ $d$ by the assumption on $\Omega$, and is not identically zero because

$$
\begin{aligned}
\langle K, g\rangle & =\int_{\mathbb{R}^{d}} \Omega(\xi)\left(\sum_{|\mathbf{i}|=1} \xi^{\mathbf{i}} \partial^{\mathbf{i}} \hat{g}(\xi)\right) d \xi=-\int_{\mathbb{R}^{d}}\left(\sum_{|\mathbf{i}|=1} \partial^{\mathbf{i}}\left(\xi^{\mathbf{i}} \Omega(\xi)\right)\right) \hat{g}(\xi) d \xi \\
& =\int_{S^{d-1}} \int_{0}^{\infty}\left(d \Omega\left(r \xi^{\prime}\right)+r \frac{d}{d r} \Omega\left(r \xi^{\prime}\right)\right) \hat{g}\left(r \xi^{\prime}\right) r^{d-1} d r d \sigma\left(\xi^{\prime}\right) \\
& =(d-\gamma) \int_{\mathbb{R}^{d}} \Omega(\xi) \hat{g}(\xi) d \xi \not \equiv 0
\end{aligned}
$$

where $g \in \mathcal{S}_{\infty}$. Thus $\int_{\|\mathbf{x}\| \geq 1}|K(\mathbf{x})|^{p} d \mathbf{x}=+\infty$ when $d<p<\infty$, and $K(\mathbf{x})$ is unbounded on $\mathbb{R}^{d} \backslash B(\mathbf{0}, 1)$ when $p=\infty$. This together with (2.42) and (2.43) proves that $J_{\Omega} \psi_{\mathbf{0}} \notin L^{p}$ and hence the desired conclusion with $f=\psi_{\mathbf{0}}$.

Case III $\gamma \geq d(1-1 / p)+1$ Let $k_{0}$ be the integer such that $d(1-1 / p) \leq$ $\gamma-k_{0}<d(1-1 / p)+1$, and set $\Omega_{\mathbf{j}}(\xi)=(i \xi)^{\mathbf{j}} \Omega(\xi),|\mathbf{j}|=k_{0}$. Noting that $J_{\Omega} \psi_{\mathbf{j}}(\mathbf{x})=J_{\Omega_{\mathbf{j}}} \psi_{\mathbf{0}}(\mathbf{x}) / \mathbf{j}$ ! and $\Omega_{\mathbf{j}}$ is homogeneous of degree $-\gamma+k_{0}$, we have obtained from the conclusions in the first two cases that $J_{\Omega} \psi_{\mathbf{j}} \notin L^{p}$. Hence the desired conclusion follows by letting $f=\psi_{\mathbf{j}}$ with $|\mathbf{j}|=k_{0}$.

\subsection{Non-integrability in the Fourier domain}

If $\gamma<d$, it follows from (2.2) that for Schwartz functions $f$ and $g,\left\langle J_{\Omega} f, g\right\rangle$ can be expressed as a weighted integral of $\hat{g}$ :

$$
\left\langle J_{\Omega} f, g\right\rangle=\int_{\mathbb{R}^{d}} h(\xi) \hat{g}(\xi) d \xi,
$$

where $h(\xi)=(2 \pi)^{-d} \Omega(-\xi) \hat{f}(-\xi) \in K_{1}$. In this subsection, we show that the above reformulation (2.44) to define $\left\langle J_{\Omega} f, g\right\rangle$ via a weighted integral of $\hat{g}$ cannot be extended to $\gamma>d$.

Theorem 2.17 Let $\gamma \in(d, \infty) \backslash \mathbb{Z}, \Omega \in C^{\infty}\left(\mathbb{R}^{d} \backslash\{\mathbf{0}\}\right)$ be a nonzero homogeneous function of degree $-\gamma$, and let $J_{\Omega}$ be defined by (2.1). Then there exists a Schwartz function $f$ such that the Fourier transform of $J_{\Omega} f$ does not belong to $K_{1}$.

Proof Let $\phi$ and $\psi_{\mathbf{0}}$ be the Schwartz functions in the proof of Lemma 2.13, and let $g \in \mathcal{S}_{\infty}$ be so chosen that its Fourier transform $\hat{g}$ is supported in $B(\mathbf{0}, 1)$ and 
satisfies $\int_{\mathbb{R}^{d}} \Omega(\xi) \hat{g}(-\xi) d \xi=1$. Now we prove that $\widehat{J_{\Omega} \psi_{0}} \notin K_{1}$. Suppose on the contrary that $\widehat{J_{\Omega} \psi_{0}} \in K_{1}$. Then

$$
\begin{aligned}
\left\langle J_{\Omega} \psi_{\mathbf{0}}, n^{-d} g(\cdot / n)\right\rangle= & \frac{(2 \pi)^{-d} \Gamma(d-\gamma)}{\Gamma\left(d+k_{0}-\gamma\right)} \int_{S^{d-1}} \int_{\epsilon}^{\infty} r^{k_{0}+d-\gamma-1} \Omega\left(\xi^{\prime}\right) \\
& \times\left(-\frac{d}{d r}\right)^{k_{0}}\left(\widehat{\psi}_{\mathbf{0}}\left(r \xi^{\prime}\right) \hat{g}\left(-r n \xi^{\prime}\right)\right) d r d \sigma\left(\xi^{\prime}\right) \\
= & (2 \pi)^{-d} \int_{\mathbb{R}^{d}} \hat{g}(-n \xi) \Omega(\xi) d \xi \\
= & (2 \pi)^{-d} n^{\gamma-d} \int_{\mathbb{R}^{d}} \Omega(\xi) \hat{g}(-\xi) d \xi \\
\rightarrow & +\infty \text { as } n \rightarrow \infty
\end{aligned}
$$

by (2.1) and (2.5). On the other hand,

$$
\begin{aligned}
& \left|\left\langle J_{\Omega} \psi_{\mathbf{0}}, n^{-d} g(\cdot / n)\right\rangle\right|=(2 \pi)^{-d}\left|\int_{\mathbb{R}^{d}} \widehat{J_{\Omega} \psi_{\mathbf{0}}}(\xi) \hat{g}(-n \xi) d \xi\right| \\
\leq & (2 \pi)^{-d}\|\hat{g}\|_{\infty} \int_{|\xi| \leq 1 / n}\left|\widehat{J_{\Omega} \psi_{0}}(\xi)\right| d \xi \rightarrow 0 \text { as } n \rightarrow \infty,
\end{aligned}
$$

where we have used the hypothesis that $\widehat{J_{\Omega} \psi_{0}} \in K_{1}$ to obtain the limit. The limits in (2.45) and (2.46) contradict each other, and hence the Fourier transform $J_{\Omega} \psi_{0}$ does not belong to $K_{1}$.

\subsection{Proof of Theorem 1.1}

Observe that $J_{\Omega}=I_{\gamma}$ when $\Omega(\xi)=\|\xi\|^{-\gamma}$ and $\gamma>0$, and that

$$
J_{\Omega}=(-\triangle)^{-\gamma / 2} \text { if } \Omega(\xi)=\|\xi\|^{-\gamma} \text { and } \gamma<0
$$

Then the necessity holds by Theorem 2.11, while the sufficiency follows from Corollary 2.3, Theorem 2.7, and Corollary 2.9.

\section{Integrable Riesz potentials}

In Section 2, we have shown that the various attempts for defining a proper (integrable) Riesz potential that is translation-invariant are doomed to failure for $\gamma>d$. We now proceed by providing a fix which is possible if we drop the translation-invariance requirement. 
Let $1 \leq p \leq \infty, \gamma \in \mathbb{R}$, and $\Omega \in C^{\infty}\left(\mathbb{R}^{d} \backslash\{\boldsymbol{0}\}\right)$ be a homogeneous function of degree $-\gamma$. We define the linear operator $U_{\Omega, p}$ from $\mathcal{S}$ to $\mathcal{S}^{\prime}$ with the help of the Fourier transform by

$$
\mathcal{F}\left(U_{\Omega, p} f\right)(\xi)=\left(\hat{f}(\xi)-\sum_{|\mathbf{i}| \leq \gamma-d(1-1 / p)} \frac{\partial^{\mathbf{i}} \hat{f}(\mathbf{0})}{\mathbf{i} !} \xi^{\mathbf{i}}\right) \Omega(\xi), \quad f \in \mathcal{S} .
$$

We call the linear operator $U_{\Omega, p}$ a p-integrable Riesz potential associated with the homogenous function $\Omega$, or integrable Riesz potential for brevity, as

$$
U_{\Omega, p}=I_{\gamma, p} \quad \text { if } \quad \Omega(\xi)=\|\xi\|^{-\gamma} .
$$

Define

$$
U_{\Omega, p}^{*} f(\mathbf{x})=(2 \pi)^{-d} \int_{\mathbb{R}^{d}}\left(e^{i(\mathbf{x}, \xi\rangle}-\sum_{|\mathbf{i}| \leq \gamma-d+d / p} \frac{(i \mathbf{x})^{\mathbf{i}} \xi}{\mathbf{i} !}\right) \Omega(-\xi) \hat{f}(\xi) d \xi, \quad f \in \mathcal{S} .
$$

Then $U_{\Omega, p}^{*}$ is the adjoint operator of the integrable Riesz potential $U_{\Omega, p}$ :

$$
\left\langle U_{\Omega, p} f, g\right\rangle=\left\langle f, U_{\Omega, p}^{*} g\right\rangle \quad \text { for all } f, g \in \mathcal{S} .
$$

If $\gamma$ satisfies $0<\gamma<d(1-1 / p)$, then

$$
U_{\Omega, p} f=J_{\Omega} f \text { for all } f \in \mathcal{S} .
$$

Hence in this case, it follows from Theorem 2.7 that $U_{\Omega, p}$ is dilation-invariant and translation-invariant, and a continuous extension of the linear operator $i_{\Omega}$ on the closed subspace $\mathcal{S}_{\infty}$ to the whole space $\mathcal{S}$. Moreover $U_{\Omega, p} f \in$ $L^{p}$ and $\mathcal{F}\left(U_{\Omega, p} f\right) \in L^{q}, 1 \leq q \leq p /(p-1)$, for any Schwartz function $f$ by Theorem 2.1 and the following estimate:

$$
\left|\mathcal{F}\left(U_{\Omega, p} f\right)(\xi)\right| \leq C\|\xi\|^{-\gamma}(1+\|\xi\|)^{\gamma-d-1} \text { for all } \xi \in \mathbb{R}^{d} .
$$

So from now on, we implicitly assume that $\gamma \geq d(1-1 / p)$, except when mentioned otherwise.

In the sequel, we investigate with the properties of the $p$-integrable Riesz potential $U_{\Omega, p}$ associated with a homogenous function $\Omega$, such as dilationinvariance and translation-variance (Theorem 3.1), $L^{p /(p-1)}$-integrability in the Fourier domain (Corollary 3.2), $L^{p}$-integrability in the spatial domain (Theorem 3.5 and Corollary 3.6), composition and left-inverse property (Theorem 3.3 and Corollary 3.4), the uniqueness of dilation-invariant extension of the linear operator $i_{\Omega}$ from the closed subspace $\mathcal{S}_{\infty}$ to the whole space $\mathcal{S}$ with additional integrability in the spatial domain and in the Fourier domain (Theorems 3.7 and 3.8). The above properties of the p-integrable 
Riesz potential associated with a homogenous function will be used to prove Theorem 1.2 in the last subsection.

3.1 Dilation-invariance, translation-variance and integrability in the Fourier domain

Theorem 3.1 Let $1 \leq p \leq \infty, \gamma \geq d(1-1 / p), k_{1}$ be the integral part of $\gamma-$ $d(1-1 / p), \Omega \in C^{\infty}\left(\mathbb{R}^{d} \backslash\{\mathbf{0}\}\right)$ be a nonzero homogeneous function of degree $-\gamma$, and let $U_{\Omega, p}$ be defined as in (3.1). Then the following statements hold.

(i) $U_{\Omega, p}$ is dilation-invariant.

(ii) $U_{\Omega, p}$ is not translation-invariant.

(iii) If $\sup _{\mathbf{x} \in \mathbb{R}^{d}}|f(\mathbf{x})|(1+\|\mathbf{x}\|)^{k_{1}+d+1+\epsilon}<\infty$ for some $\epsilon>0$, then there exists a positive constant $C$ independent on $f$ such that

$$
\left|\mathcal{F}\left(U_{\Omega, p} f\right)(\xi)\right| \leq C\left(\sup _{\mathbf{z} \in \mathbb{R}^{d}}|f(\mathbf{z})|(1+\|\mathbf{z}\|)^{k_{1}+d+1+\epsilon}\right)\|\xi\|^{k_{1}-\gamma+1}(1+\|\xi\|)^{-1}
$$

for all $\xi \in \mathbb{R}^{d}$.

(iv) $U_{\Omega, p}$ is a continuous linear operator from $\mathcal{S}$ to $\mathcal{S}^{\prime}$, and an extension of the operator $i_{\Omega}$ on the subspace $\mathcal{S}_{\infty}$ to the whole space $\mathcal{S}$.

As a consequence of Theorem 3.1, we have the following result about the $L^{p /(p-1)}$-integrability of the Fourier transform of $U_{\Omega, p} f$ for $f \in \mathcal{S}$.

Corollary 3.2 Let $1 \leq p \leq \infty$ and $\gamma \geq d(1-1 / p)$ satisfy either $p=1$ or $\gamma-$ $d(1-1 / p) \notin \mathbb{Z}_{+}$and $1<p \leq \infty, k_{1}$ be the integral part of $\gamma-d(1-1 / p), \Omega \in$ $C^{\infty}\left(\mathbb{R}^{d} \backslash\{\mathbf{0}\}\right)$ be a homogeneous function of degree $-\gamma$, and let $U_{\Omega, p}$ be defined as in (3.1). Then the Fourier transform of $U_{\Omega, p} f$ belongs to $L^{p /(p-1)}$ for any $f \in \mathcal{S}$.

Proof of Theorem 3.1 (i) Given any $t>0$ and $f \in \mathcal{S}$,

$$
\begin{aligned}
\mathcal{F}\left(U_{\Omega, p}\left(\delta_{t} f\right)\right)(\xi) & =t^{-d}\left(\hat{f}\left(\frac{\xi}{t}\right)-\sum_{|\mathbf{i}| \leq \gamma-d+d / p} \frac{\partial^{\mathbf{i}} \hat{f}(\mathbf{0})}{\mathbf{i} !}\left(\frac{\xi}{t}\right)^{\mathbf{i}}\right) \Omega(\xi) \\
& =t^{-d-\gamma} \mathcal{F}\left(U_{\Omega, p} f\right)\left(\frac{\xi}{t}\right) .
\end{aligned}
$$

This proves the dilation-invariance of the linear operator $U_{\Omega, p}$.

(ii) Suppose, on the contrary, that $U_{\Omega, p}$ is translation-invariant. Then

$$
\Omega(\xi) \sum_{|\mathbf{i}| \leq \gamma-d+d / p} \frac{\partial^{\mathbf{i}} \widehat{\tau_{\mathbf{x}_{0}} f(\mathbf{0})}}{\mathbf{i} !} \xi^{\mathbf{i}}=\Omega(\xi) e^{-i\left|\mathbf{x}_{0}, \xi\right\rangle} \sum_{|\mathbf{i}| \leq \gamma-d+d / p} \frac{\partial^{\mathbf{i}} \hat{f}(\mathbf{0})}{\mathbf{i} !} \xi^{\mathbf{i}}, \quad \xi \in \mathbb{R}^{d}
$$


for all $\mathbf{x}_{0} \in \mathbb{R}^{d}$ and $f \in \mathcal{S}$. Note that the left-hand side of equation (3.7) is a polynomial in $\mathbf{x}_{0}$ by (2.32) while its right hand side is a trigonometric function of $\mathbf{x}_{0}$. Hence both sides must be identically zero, which implies that

$$
\Omega(\xi) \sum_{|\mathbf{i}| \leq \gamma-d+d / p} \frac{\partial^{\mathbf{i}} \hat{f}(\mathbf{0})}{\mathbf{i} !} \xi^{\mathbf{i}}=0, \quad \xi \in \mathbb{R}^{d}
$$

for all $f \in \mathcal{S}$. Replacing $f$ in the above equation by the function $\psi_{0}$ in (2.29) and using (2.30) and the assumption $\gamma \geq d(1-1 / p)$ leads to a contradiction. (iii) By the assumption on the homogeneous function $\Omega,|\Omega(\xi)| \leq C\|\xi\|^{-\gamma}$. Then for $\xi \in \mathbb{R}^{d}$ with $\|\xi\| \geq 1$,

$$
\begin{aligned}
\left|\mathcal{F}\left(U_{\Omega, p} f\right)(\xi)\right| & \leq C\left(\|\hat{f}\|_{\infty}+\sum_{|\mathbf{i}| \leq k_{1}}\left\|\partial^{\mathbf{i}} \hat{f}\right\|_{\infty}\|\xi\|^{|\mathbf{i}|}\right)\|\xi\|^{-\gamma} \\
& \leq C\left(\sum_{|\mathbf{i}| \leq k_{1}+1}\left\|\partial^{\mathbf{i}} \hat{f}\right\|_{\infty}\right)\|\xi\|^{k_{1}-\gamma}
\end{aligned}
$$

by (3.1), and for $\xi \in \mathbb{R}^{d}$ with $\|\xi\| \leq 1$,

$$
\left|\mathcal{F}\left(U_{\Omega, p} f\right)(\xi)\right| \leq C\left(\sum_{|\mathbf{i}| \leq k_{1}+1}\left\|\partial^{\mathbf{i}} \hat{f}\right\|_{\infty}\right)\|\xi\|^{k_{1}-\gamma+1}
$$

by the Taylor's expansion to the function $\hat{f}(\xi)$ at the origin. Combining the above two estimates gives

$$
\left|\mathcal{F}\left(U_{\Omega, p} f\right)(\xi)\right| \leq C\left(\sum_{|\mathbf{i}| \leq k_{1}+1}\left\|\partial^{\mathbf{i}} \hat{f}\right\|_{\infty}\right)\|\xi\|^{k_{1}-\gamma+1}(1+\|\xi\|)^{-1}, \quad \xi \in \mathbb{R}^{d} .
$$

Note that

$$
\left\|\partial^{\mathbf{i}} \hat{f}\right\|_{\infty} \leq C \int_{\mathbb{R}^{d}}|f(\mathbf{x})||\mathbf{x}|^{\mid \mathbf{i}} d \mathbf{x} \leq C \sup _{\mathbf{z} \in \mathbb{R}^{d}}|f(\mathbf{z})|(1+|\mathbf{z}|)^{k_{1}+d+1+\epsilon}
$$

for all $\mathbf{i} \in \mathbb{Z}_{+}^{d}$ with $|\mathbf{i}| \leq k_{1}+1$. Then the desired estimate (3.6) follows from (3.9) and (3.10).

(iv) By (3.1) and the first conclusion of this theorem, the Fourier transform of $U_{\Omega, p} f$ is continuous on $\mathbb{R}^{d} \backslash\{\mathbf{0}\}$, and satisfies

$$
\int_{\mathbb{R}^{d}}\left|\mathcal{F}\left(U_{\Omega, p} f\right)(\xi)\right|(1+\|\xi\|)^{\gamma-k_{1}-d-1} d \xi \leq C \sup _{\mathbf{z} \in \mathbb{R}^{d}}|f(\mathbf{x})|(1+\|\mathbf{x}\|)^{k_{1}+d+2} .
$$

Hence $U_{\Omega, p}$ is a continuous linear operator from $\mathcal{S}$ to $\mathcal{S}^{\prime}$. For any $f \in \mathcal{S}_{\infty}$, $\partial^{\mathbf{i}} \hat{f}(\mathbf{0})=0$ for all $\mathbf{i} \in \mathbb{Z}_{+}^{d}$. Then $\mathcal{F}\left(U_{\Omega, p} f\right)=\mathcal{F}\left(i_{\Omega} f\right)$ for all $f \in \mathcal{S}_{\infty}$. This shows that $U_{\Omega, p}, 1 \leq p \leq \infty$, is a continuous extension of the linear operator $i_{\Omega}$ from the subspace $\mathcal{S}_{\infty} \subset \mathcal{S}$ to the whole space $\mathcal{S}$. 
3.2 Composition and left-inverse of the fractional Laplacian

Direct calculation leads to

$$
\sum_{|\mathbf{i}| \leq \gamma-d(1-1 / p)} \frac{\partial^{\left.\mathbf{i}\left(\xi^{\mathbf{k}} \hat{f}(\xi)\right)\right|_{\xi=\mathbf{0}}}}{\mathbf{i} !} \xi^{\mathbf{i}}=\sum_{|\mathbf{j}| \leq \gamma-|\mathbf{k}|-d(1-1 / p)} \frac{\partial^{\mathbf{j}} \hat{f}(\mathbf{0})}{\mathbf{j} !} \xi^{\mathbf{j}+\mathbf{k}}, \quad \mathbf{k} \in \mathbb{Z}_{+}^{d}
$$

for any $\gamma \in \mathbb{R}, 1 \leq p \leq \infty$ and $f \in \mathcal{S}$. This together with (3.1) implies that

$$
U_{\Omega, p}\left(\partial^{\mathbf{k}} f\right)=U_{\Omega_{\mathbf{k}}, p} f \quad \text { for all } f \in \mathcal{S} \text { and } \mathbf{k} \in \mathbb{Z}_{+}^{d},
$$

where $\Omega_{\mathbf{k}}(\xi)=(i \xi)^{\mathbf{k}} \Omega(\xi)$ for $\mathbf{k} \in \mathbb{Z}_{+}^{d}$. In general, we have the following result about composition of integrable Riesz potentials.

Theorem 3.3 Let $1 \leq p \leq \infty$, real numbers $\gamma_{1}, \gamma_{2}$ satisfy $\gamma_{1} \geq d(1-1 / p)$ and $-\gamma_{2}$ is larger than the integral part of $\gamma_{1}-d(1-1 / p)$, and let $\Omega_{1}, \Omega_{2} \in$ $C^{\infty}\left(\mathbb{R}^{d} \backslash\{0\}\right)$ be homogenous of degree $-\gamma_{1}$ and $-\gamma_{2}$ respectively. Then

$$
U_{\Omega_{1}, p}\left(J_{\Omega_{2}} f\right)=J_{\Omega_{1} \Omega_{2}} f \text { for all } f \in \mathcal{S} .
$$

As a consequence of Theorems 2.8 and 3.3, we have the following result about the left-inverse of the fractional Laplacian $(-\Delta)^{\gamma / 2}$.

Corollary 3.4 Let $1 \leq p \leq \infty$ and $\gamma>0$ satisfy either $1<p \leq \infty$ or $p=1$ and $\gamma \notin \mathbb{Z}_{+}$, and the linear operator $I_{\gamma, p}$ be defined as in (1.12). Then $I_{\gamma, p}$ is a leftinverse of the fractional Laplacian $(-\triangle)^{\gamma / 2}$, i.e., $I_{\gamma, p}(-\triangle)^{\gamma / 2} f=f$ for all $f \in \mathcal{S}$.

Proof of Theorem 3.3 Let $k_{1}$ be the integral part of $\gamma_{1}-d(1-1 / p)$. Then $-\gamma_{2}>k_{1}$ by the assumption. Then $\mathcal{F}\left(J_{\Omega_{2}} f\right)(\xi)=\Omega_{2}(\xi) \hat{f}(\xi)$ and $\left.\partial^{\mathbf{i}}\left(\mathcal{F}\left(J_{\Omega_{2}} f\right)(\xi)\right)\right|_{\xi=\mathbf{0}}=0$ for any $\mathbf{i} \in \mathbb{Z}_{+}$with $|\mathbf{i}| \leq k_{1}$ and any Schwartz function $f$. This implies that $\mathcal{F}\left(U_{\Omega_{1}, p}\left(J_{\Omega_{2}} f\right)\right)(\xi)$ is equal to

$$
\left(\widehat{J_{\Omega_{2}} f}(\xi)-\sum_{|\mathbf{i}| \leq \gamma_{1}-d(1-1 / p)} \frac{\left.\partial^{\mathbf{i}}\left(\mathcal{F}\left(J_{\Omega_{2}} f\right)(\xi)\right)\right|_{\xi=\mathbf{0}}}{\mathbf{i} !} \xi^{\mathbf{i}}\right) \Omega_{1}(\xi),
$$

which is the same as $\mathcal{F}\left(J_{\Omega_{1} \Omega_{2}} f\right)(\xi)$. Hence the equation (3.12) is established.

\section{$3.3 L^{p}$-integrability in the spatial domain}

If $\gamma \in(0, d(1-1 / p))$, then it follows from (3.1) and Theorem 2.1 that $\left|U_{\Omega, p} f(\mathbf{x})\right| \leq C(1+\|\mathbf{x}\|)^{\gamma-d}, \quad \mathbf{x} \in \mathbb{R}^{d}$ (hence $U_{\Omega, p} f \in L^{p}$ ) for any Schwartz function $f$. In this subsection, we provide a similar estimate for $U_{\Omega, p} f$ when $\gamma \geq d(1-1 / p)$. 
Theorem 3.5 Let $0<\epsilon<1,1 \leq p \leq \infty, \gamma \in[d(1-1 / p), \infty) \backslash \mathbb{Z}, k_{1}$ be the integral part of $\gamma-d(1-1 / p)$, and $\Omega \in C^{\infty}\left(\mathbb{R}^{d} \backslash\{\mathbf{0}\}\right)$ be a homogeneous function of degree $-\gamma$. If

$$
|f(\mathbf{x})| \leq C(1+\|\mathbf{x}\|)^{-\left(k_{1}+1+d+\epsilon\right)}, \quad \mathbf{x} \in \mathbb{R}^{d}
$$

then

$$
\begin{aligned}
\left|U_{\Omega, p} f(\mathbf{x})\right| \leq & C\left(\sup _{\mathbf{z} \in \mathbb{R}^{d}}|f(\mathbf{z})|(1+\|\mathbf{z}\|)^{k_{1}+1+d+\epsilon}\right) \\
& \times\|\mathbf{x}\|^{\min \left(\gamma-k_{1}-d, 0\right)}(1+\|\mathbf{x}\|)^{\max \left(\gamma-k_{1}-d, 0\right)-1}
\end{aligned}
$$

for all $\mathbf{x} \in \mathbb{R}^{d}$, and

$$
\begin{aligned}
\left|U_{\Omega, p} f(\mathbf{x})-U_{\Omega, p} f\left(\mathbf{x}^{\prime}\right)\right| \leq & C\left(\sup _{\mathbf{z} \in \mathbb{R}^{d}}|f(\mathbf{z})|(1+\|\mathbf{z}\|)^{k_{1}+1+d+\epsilon}\right)\left\|\mathbf{x}-\mathbf{x}^{\prime}\right\|^{\delta} \\
& \times\|\mathbf{x}\|^{\min \left(\gamma-k_{1}-d-\delta, 0\right)}(1+\|\mathbf{x}\|)^{\max \left(\gamma-k_{1}-d-\delta, 0\right)-1}
\end{aligned}
$$

for all $\mathbf{x}, \mathbf{x}^{\prime} \in \mathbb{R}^{d}$ with $\left\|\mathbf{x}-\mathbf{x}^{\prime}\right\| \leq\|\mathbf{x}\| / 4$, where $\delta<\min \left(\left|\gamma-k_{1}-d\right|, \epsilon\right)$.

As an easy consequence of Theorem 3.5, we have

Corollary 3.6 Let $1 \leq p \leq \infty, \gamma \geq d(1-1 / p)$, and $\Omega \in C^{\infty}\left(\mathbb{R}^{d} \backslash\{\mathbf{0}\}\right)$ be a homogeneous function of degree $\gamma$. If both $\gamma$ and $\gamma-d(1-1 / p)$ are not nonnegative integers, then $U_{\Omega, p} f$ is Hölder continuous on $\mathbb{R}^{d} \backslash\{\mathbf{0}\}$ and belong to $L^{p}$ for any Schwartz function $f$.

Proof of Theorem 3.5 We investigate three cases to establish the estimates in (3.14) and (3.15).

Case I $k_{1}+1-\gamma<0 \quad \operatorname{Set} h_{\xi}(t)=\hat{f}(t \xi)$. Applying Taylor's expansion to the function $h_{\xi}$ gives

$$
\begin{aligned}
\hat{f}(\xi) & =h_{\xi}(1)=\sum_{s=0}^{k_{1}} \frac{h^{(s)}(0)}{s !}+\frac{1}{k_{1} !} \int_{0}^{1} h_{\xi}^{\left(k_{1}+1\right)}(t)(1-t)^{k_{1}} d t \\
& =\sum_{|\mathbf{i}| \leq k_{1}} \frac{\partial^{\mathbf{i}} \hat{f}(\mathbf{0})}{\mathbf{i} !} \xi^{\mathbf{i}}+\left(k_{1}+1\right) \sum_{|\mathbf{j}|=k_{1}+1} \frac{\xi^{\mathbf{j}}}{\mathbf{j} !} \int_{0}^{1} \partial^{\mathbf{j}} \hat{f}(t \xi)(1-t)^{k_{1}} d t .
\end{aligned}
$$

Hence

$$
\left(\hat{f}(\xi)-\sum_{|\mathbf{i}| \leq k_{1}} \frac{\partial^{\mathbf{i}} \hat{f}(\mathbf{0})}{\mathbf{i} !} \xi^{\mathbf{i}}\right) \Omega(\xi)=\sum_{|\mathbf{j}|=k_{1}+1} \frac{1}{\mathbf{j} !} \Omega_{\mathbf{j}}(\xi) \widehat{g}_{\mathbf{j}}(\xi),
$$

where $\Omega_{\mathbf{j}}(\xi)=(i \xi)^{\mathbf{j}} \Omega(\xi)$ and

$$
g_{\mathbf{j}}(\mathbf{x})=\left(k_{1}+1\right) \int_{0}^{1}(1-t)^{k_{1}}(-\mathbf{x} / t)^{\mathbf{j}} f(\mathbf{x} / t) t^{-d} d t \in L^{1}, \quad|\mathbf{j}|=k_{1}+1 .
$$


Taking inverse Fourier transform at both sides of the equation (3.17) yields

$$
U_{\Omega, p} f(\mathbf{x})=\sum_{|\mathbf{j}|=k_{1}+1} \frac{1}{\mathbf{j} !} \int_{\mathbb{R}^{d}} K_{\mathbf{j}}(\mathbf{x}-\mathbf{y}) g_{\mathbf{j}}(\mathbf{y}) d \mathbf{y}
$$

where $K_{\mathbf{j}},|\mathbf{j}|=k_{1}+1$, is the inverse Fourier transform of $\Omega_{\mathbf{j}}$. Therefore

$$
\begin{aligned}
\left|U_{\Omega, p} f(\mathbf{x})\right| \leq & C \int_{0}^{1} \int_{\mathbb{R}^{d}}\|\mathbf{x}-\mathbf{y}\|^{\gamma-d-k_{1}-1}\|\mathbf{y} / t\|^{k_{1}+1}|f(\mathbf{y} / t)| t^{-d} d \mathbf{y} d t \\
= & C \int_{0}^{1} \int_{\mathbb{R}^{d}}\|\mathbf{x}-t \mathbf{y}\|^{\gamma-d-k_{1}-1}\|\mathbf{y}\|^{k_{1}+1}|f(\mathbf{y})| d \mathbf{y} d t \\
\leq & C\left(\sup _{\mathbf{z} \in \mathbb{R}^{d}}|f(\mathbf{z})|(1+\|\mathbf{z}\|)^{k_{1}+1+d+\epsilon}\right) \int_{0}^{1}(t+\|\mathbf{x}\|)^{\gamma-d-k_{1}-1} d t \\
\leq & C\left(\sup _{\mathbf{z} \in \mathbb{R}^{d}}|f(\mathbf{z})|(1+\|\mathbf{z}\|)^{k_{1}+1+d+\epsilon}\right) \\
& \times\|\mathbf{x}\|^{\min \left(\gamma-d-k_{1}, 0\right)}(1+\|\mathbf{x}\|)^{\max \left(\gamma-d-k_{1}, 0\right)-1},
\end{aligned}
$$

where the first inequality holds because $K_{\mathbf{j}} \in C^{\infty}\left(\mathbb{R}^{d} \backslash\{\boldsymbol{0}\}\right)$ is homogeneous of degree $\gamma-d-k_{1}-1 \in(-d, 0)$ [7, Theorems 7.1.16 and 7.1.18], and the second inequality follows from (2.10). Similarly,

$$
\begin{aligned}
& \left|U_{\Omega, p} f(\mathbf{x})-U_{\Omega, p} f\left(\mathbf{x}^{\prime}\right)\right| \\
& \leq C \sum_{|\mathbf{j}|=k_{1}+1} \int_{\|\mathbf{x}-\mathbf{y}\| \geq 2\left\|\mathbf{x}-\mathbf{x}^{\prime}\right\|}\left\|\mathbf{x}-\mathbf{x}^{\prime}\right\|^{\delta}\|\mathbf{x}-\mathbf{y}\|^{\gamma-d-k_{1}-1-\delta}\left|g_{\mathbf{j}}(\mathbf{y})\right| d \mathbf{y} \\
& \quad+C \sum_{|\mathbf{j}|=k_{1}+1} \int_{\|\mathbf{x}-\mathbf{y}\| \leq 2\left\|\mathbf{x}-\mathbf{x}^{\prime}\right\|}\|\mathbf{x}-\mathbf{y}\|^{\gamma-d-k_{1}-1}\left|g_{\mathbf{j}}(\mathbf{y})\right| d \mathbf{y} \\
& \quad+C \sum_{|\mathbf{j}|=k_{1}+1} \int_{\|\mathbf{x}-\mathbf{y}\| \leq 2\left\|\mathbf{x}-\mathbf{x}^{\prime}\right\|}\left\|\mathbf{x}^{\prime}-\mathbf{y}\right\|^{\gamma-d-k_{1}-1}\left|g_{\mathbf{j}}(\mathbf{y})\right| d \mathbf{y} \\
& \leq C\left(\sup _{\mathbf{z} \in \mathbb{R}^{d}}|f(\mathbf{z})|(1+\|\mathbf{z}\|)^{k_{1}+1+d+\epsilon}\right)\left\|\mathbf{x}-\mathbf{x}^{\prime}\right\|^{\delta} \\
& \quad \times\|\mathbf{x}\|^{\min \left(\gamma-d-k_{1}-\delta, 0\right)}(1+\|\mathbf{x}\|)^{\max \left(\gamma-d-k_{1}-\delta, 0\right)-1}
\end{aligned}
$$

for all $\mathbf{x}, \mathbf{x}^{\prime} \in \mathbb{R}^{d}$ with $\left\|\mathbf{x}-\mathbf{x}^{\prime}\right\| \leq\|\mathbf{x}\| / 4$, where $\delta<\min \left(\epsilon,\left|\gamma-k_{1}-d\right|\right)$. Then the desired estimate (3.14) and (3.15) follow from (3.20) and (3.21) for the case $k_{1}+1-\gamma<0$. 
Case II $k_{1}+1-\gamma>0$ and $k_{1} \geq 1 \quad$ Applying Taylor's expansion to the function $h_{\xi}(t)=\hat{f}(t \xi)$, we have

$$
\hat{f}(\xi)-\sum_{|\mathbf{i}| \leq k_{1}} \frac{\partial^{\mathbf{i}} \hat{f}(\mathbf{0})}{\mathbf{i} !} \xi^{\mathbf{i}}=k_{1} \sum_{|\mathbf{j}|=k_{1}} \frac{\xi^{\mathbf{j}}}{\mathbf{j} !} \int_{0}^{1}\left(\partial^{\mathbf{j}} \hat{f}(t \xi)-\partial^{\mathbf{j}} \hat{f}(\mathbf{0})\right)(1-t)^{k_{1}-1} d t
$$

Multiplying by $\Omega(\xi)$ both sides of the above equation and then taking the inverse Fourier transform, we obtain

$$
U_{\Omega, p} f(\mathbf{x})=\sum_{|\mathbf{j}|=k_{1}} \frac{1}{\mathbf{j} !}\left(\int_{\mathbb{R}^{d}} K_{\mathbf{j}}(\mathbf{x}-\mathbf{y}) g_{\mathbf{j}}(\mathbf{y}) d \mathbf{y}-K_{\mathbf{j}}(\mathbf{x}) \int_{\mathbb{R}^{d}} g_{\mathbf{j}}(\mathbf{y}) d \mathbf{y}\right),
$$

where

$$
g_{\mathbf{j}}(\mathbf{x})=k_{1} \int_{0}^{1}(1-t)^{k_{1}-1}(-\mathbf{x} / t)^{\mathbf{j}} f(\mathbf{x} / t) t^{-d} d t \in L^{1}, \quad|\mathbf{j}|=k_{1}
$$

Recalling that $K_{\mathbf{j}} \in C^{\infty}\left(\mathbb{R}^{d} \backslash\{\mathbf{0}\}\right),|\mathbf{j}|=k_{1}$ are homogeneous of degree $\gamma-d-$ $k_{1} \in(-d, 0)$,

$$
\left|\partial^{\mathbf{i}} K_{\mathbf{j}}(\mathbf{x})\right| \leq C\|\mathbf{x}\|^{\gamma-d-k_{1}-|\mathbf{j}|}, \quad|\mathbf{i}| \leq 1
$$

Combining (2.10), (3.22), (3.23) and (3.24), we get

$$
\begin{aligned}
\left|U_{\Omega, p} f(\mathbf{x})\right| \leq & C \sum_{|\mathbf{j}|=k_{1}} \int_{0}^{1} \int_{\mathbb{R}^{d}}\left|K_{\mathbf{j}}(\mathbf{x}-t \mathbf{y})-K_{\mathbf{j}}(\mathbf{x})\right|\|\mathbf{y}\|^{k_{1}}|f(\mathbf{y})| d \mathbf{y} \\
\leq & C\left(\sup _{\mathbf{z} \in \mathbb{R}^{d}}|f(\mathbf{z})|(1+\|\mathbf{z}\|)^{k_{1}+d+1+\epsilon}\right) \\
& \times\left\{\int_{0}^{1} \int_{\|\mathbf{y}\| \leq\|\mathbf{x}\| / 2} t\|\mathbf{y}\|\|\mathbf{x}\|^{\gamma-d-k_{1}-1}(1+\|\mathbf{y}\|)^{-d-1-\epsilon} d \mathbf{y} d t\right. \\
& +(1+\|\mathbf{x}\|)^{-1} \int_{0}^{1} \int_{\|\mathbf{y}\| \geq\|\mathbf{x}\| / 2}\|\mathbf{x}-t \mathbf{y}\|^{\gamma-d-k_{1}}(1+\|\mathbf{y}\|)^{-d-\epsilon} d \mathbf{y} d t \\
& \left.+\|\mathbf{x}\|^{\gamma-d-k_{1}} \int_{0}^{1} \int_{\|\mathbf{y}\| \geq\|\mathbf{x}\| / 2}(1+\|\mathbf{y}\|)^{-d-1-\epsilon} d \mathbf{y} d t\right\} \\
\leq & C\left(\sup _{\mathbf{z} \in \mathbb{R}^{d}}|f(\mathbf{z})|(1+\|\mathbf{z}\|)^{k_{1}+d+1+\epsilon}\right) \\
& \times\|\mathbf{x}\|^{\min \left(\gamma-k_{1}-d, 0\right)}(1+\|\mathbf{x}\|)^{\max \left(\gamma-k_{1}-d, 0\right)-1}
\end{aligned}
$$


and

$$
\begin{array}{rl}
\mid U_{\Omega, p} & f(\mathbf{x})-U_{\Omega, p} f\left(\mathbf{x}^{\prime}\right) \mid \\
\leq & C \sum_{|\mathbf{j}|=k_{1}} \int_{0}^{1}\left(\int_{\|\mathbf{y}\| \leq\|\mathbf{x}\| / 4}+\int_{\|\mathbf{y}\| \geq 4\|\mathbf{x}\|}+\int_{\|\mathbf{x}\| / 4 \leq\|t \mathbf{y}\| \leq 4\|\mathbf{x}\|}\right) \\
& \left|K_{\mathbf{j}}(\mathbf{x}-t \mathbf{y})-K_{\mathbf{j}}(\mathbf{x})-K_{\mathbf{j}}\left(\mathbf{x}^{\prime}-t \mathbf{y}\right)+K_{\mathbf{j}}\left(\mathbf{x}^{\prime}\right)\right|\|\mathbf{y}\|^{k_{1}}|f(\mathbf{y})| d \mathbf{y} \\
\leq & C\left(\sup _{\mathbf{z} \in \mathbb{R}^{d}}|f(\mathbf{z})|(1+\|\mathbf{z}\|)^{k_{1}+d+1+\epsilon}\right) \sum_{|\mathbf{j}|=k_{1}}\left\{\left\|\mathbf{x}-\mathbf{x}^{\prime}\right\|^{\delta}\right. \\
\quad & \int_{0}^{1} \int_{\|\mathbf{y}\| \leq\|\mathbf{x}\| / 4} t\|\mathbf{y}\|\|\mathbf{x}\|^{\gamma-d-k_{1}-1-\delta}(1+\|\mathbf{y}\|)^{-d-1-\epsilon} d \mathbf{y} d t+\left\|\mathbf{x}-\mathbf{x}^{\prime}\right\|^{\delta} \\
& \times \int_{0}^{1} \int_{t\|\mathbf{y}\| \geq 4\|\mathbf{x}\|}\left(\|\mathbf{x}\|^{\gamma-k_{1}-d-\delta}+\|\mathbf{y}\|^{\gamma-k_{1}-d-\delta}\right)(1+\|\mathbf{y}\|)^{-d-1-\epsilon} d \mathbf{y} d t \\
& +\int_{0}^{1} \int_{\|\mathbf{x}\| / 4 \leq\|\mathbf{y}\| \leq 4\|\mathbf{x}\|}\left(\left|K_{\mathbf{j}}(\mathbf{x}-t \mathbf{y})-K_{\mathbf{j}}\left(\mathbf{x}^{\prime}-t \mathbf{y}\right)\right|+\left|K_{\mathbf{j}}(\mathbf{x})-K_{\mathbf{j}}(\mathbf{x})\right|\right) \\
& \left.\times(1+\|\mathbf{x}\| / t)^{-d-1-\epsilon} d \mathbf{y} d t\right\} \\
\leq & C\left(\sup _{\mathbf{z} \in \mathbb{R}^{d}}|f(\mathbf{z})|(1+\|\mathbf{z}\|)^{k_{1}+d+1+\epsilon}\right)\left\|\mathbf{x}-\mathbf{x}^{\prime}\right\|^{\delta}\|\mathbf{x}\|^{\gamma-k_{1}-d-\delta}(1+\|\mathbf{x}\|)^{-1} .
\end{array}
$$

Then the desired estimates (3.14) and (3.15) are proved in the case that $k_{1}+$ $1-\gamma>0$ and $k_{1} \geq 1$.

Case III $k_{1}+1-\gamma>0$ and $k_{1}=0$ In this case, $\gamma \in(0,1)$ and

$$
U_{\Omega, p} f(\mathbf{x})=\int_{\mathbb{R}^{d}}(K(\mathbf{x}-\mathbf{y})-K(\mathbf{x})) f(\mathbf{y}) d \mathbf{y}
$$

where $K$ is the inverse Fourier transform of $\Omega(\xi)$. Then, by applying the argument used in establishing (3.25), we have

$$
\begin{aligned}
\left|U_{\Omega, p} f(\mathbf{x})\right| \leq & C\left(\sup _{\mathbf{z} \in \mathbb{R}^{d}}|f(\mathbf{z})|(1+\|\mathbf{z}\|)^{d+1+\epsilon}\right) \\
& \times\left\{\int_{\|\mathbf{y}\| \leq\|\mathbf{x}\| / 2} t\|\mathbf{y}\|\|\mathbf{x}\|^{\gamma-d-1}(1+\|\mathbf{y}\|)^{-d-1-\epsilon} d \mathbf{y}\right. \\
& +(1+\|\mathbf{x}\|)^{-1} \int_{\|\mathbf{y}\| \geq\|\mathbf{x}\| / 2}\|\mathbf{x}-\mathbf{y}\|^{\gamma-d}(1+\|\mathbf{y}\|)^{-d-\epsilon} d \mathbf{y} \\
& \left.+\|\mathbf{x}\|^{\gamma-d} \int_{\|\mathbf{y}\| \geq\|\mathbf{x}\| / 2}(1+\|\mathbf{y}\|)^{-d-1-\epsilon} d \mathbf{y}\right\} \\
\leq & C\left(\sup _{\mathbf{z} \in \mathbb{R}^{d}}|f(\mathbf{z})|(1+\|\mathbf{z}\|)^{d+1+\epsilon}\right)\|\mathbf{x}\|^{\gamma-d}(1+\|\mathbf{x}\|)^{-1},
\end{aligned}
$$


and

$$
\begin{aligned}
& \left|U_{\Omega, p} f(\mathbf{x})-U_{\Omega, p} f\left(\mathbf{x}^{\prime}\right)\right| \\
\leq & \left(\int_{\|\mathbf{y}\| \leq\|\mathbf{x}\| / 4}+\int_{\|\mathbf{y}\| \geq 4\|\mathbf{x}\|}+\int_{\|\mathbf{x}\| / 4 \leq\|\mathbf{y}\| \leq 4\|\mathbf{x}\|}\right) \\
& \left|K(\mathbf{x}-\mathbf{y})-K(\mathbf{x})-K\left(\mathbf{x}^{\prime}-\mathbf{y}\right)+K\left(\mathbf{x}^{\prime}\right) \| f(\mathbf{y})\right| d \mathbf{y} \\
\leq & C\left(\sup _{\mathbf{z} \in \mathbb{R}^{d}}|f(\mathbf{z})|(1+\|\mathbf{z}\|)^{k_{1}+d+1+\epsilon}\right)\left\|\mathbf{x}-\mathbf{x}^{\prime}\right\|^{\delta}\|\mathbf{x}\|^{\gamma-d-\delta}(1+\|\mathbf{x}\|)^{-1},
\end{aligned}
$$

which yields the desired estimates (3.14) and (3.15) for $k_{1}+1-\gamma>0$ and $k_{1}=0$.

3.4 Unique dilation-invariant extension of the linear operator $i_{\Omega}$ with additional integrability in the spatial domain

We now show that $U_{\Omega, p}$ is the only dilation-invariant extension of the linear operator $i_{\Omega}$ from the subspace $\mathcal{S}_{\infty}$ to the whole space $\mathcal{S}$ such that its image is contained in $L^{p}$.

Theorem 3.7 Let $1 \leq p \leq \infty, \gamma>0$ have the property that both $\gamma$ and $\gamma-$ $d(1-1 / p)$ are not nonnegative integers, $\Omega \in C^{\infty}\left(\mathbb{R}^{d} \backslash\{\mathbf{0}\}\right)$ be a nonzero homogeneous function of degree $-\gamma$, and the linear map I from $\mathcal{S}$ to $\mathcal{S}^{\prime}$ be a homogeneous extension of the linear operator $i_{\Omega}$ on $\mathcal{S}_{\infty}$. Then If belongs to $L^{p}$ for any Schwartz function $f$ if and only if $I=U_{\Omega, p}$.

Proof The sufficiency follows from (3.1) and Theorems 1.1 and 2.1 for $\gamma<$ $d(1-1 / p)$, and from (3.1), Theorem 3.1 and Corollary 3.6 for $\gamma \geq d(1-1 / p)$. Now the necessity. By the assumption on the linear operator $I$ from $\mathcal{S}$ to $\mathcal{S}^{\prime}$, similar to the argument used in Lemma 2.12, we can find an integer $N$ and tempered distributions $H_{\mathbf{i}},|\mathbf{i}| \leq N$, such that

$$
I f=U_{\Omega, p} f+\sum_{|\mathbf{i}| \leq N} \frac{\partial^{\mathbf{i}} \hat{f}(\mathbf{0})}{\mathbf{i} !} H_{\mathbf{i}} \quad \text { for all } f \in \mathcal{S} .
$$

Replacing $f$ in (3.30) by $\psi_{\mathbf{j}}$ in (2.29) and using (2.30) gives that $H_{\mathbf{j}} / \mathbf{j} !=I \psi_{\mathbf{j}}-$ $U_{\Omega, p} \psi_{\mathbf{j}}$. Hence

$$
H_{\mathbf{j}} \in L^{p}
$$

by Corollary 3.6 and the assumption on the linear map I. By (3.30), Theorem 3.1 and the assumption on the linear operator $I,\left(I-U_{\Omega, p}\right)\left(\delta_{t} f\right)=$ $t^{-\gamma} \delta_{t}\left(\left(I-U_{\Omega, p}\right) f\right)$ for all $f \in \mathcal{S}$. Hence $H_{\mathbf{j}}$ is homogeneous of order $\gamma-d-|\mathbf{j}|$ by Lemma 2.13. This together with (3.31) implies that $H_{\mathbf{j}}=0$ for all $\mathbf{j} \in \mathbb{Z}_{+}^{d}$ with $|\mathbf{j}| \leq N$. The desired conclusion $I=U_{\Omega, p}$ then follows. 
3.5 Unique dilation-invariant extension of the linear operator $i_{\Omega}$ with additional integrability in the Fourier domain

In this subsection, we characterize all those dilation-invariant extensions $I$ of the linear operator $i_{\Omega}$ on the subspace $\mathcal{S}_{\infty}$ to the whole space $\mathcal{S}$ such that $\widehat{I f}$ is $q$-integrable for any Schwartz function $f$.

Theorem 3.8 Let $1 \leq q \leq \infty, \gamma \in[d / q, \infty) \backslash \mathbb{Z}$ and $\Omega \in C^{\infty}\left(\mathbb{R}^{d} \backslash\{\mathbf{0}\}\right)$ be a nonzero homogeneous function of degree $-\gamma$, and the linear map I from $\mathcal{S}$ to $\mathcal{S}^{\prime}$ be a dilation-invariant extension of the linear operator $i_{\Omega}$ on $\mathcal{S}_{\infty}$. Then the following statements hold.

(i) If $1 \leq q<\infty$, then the Fourier transform of If belongs to $L^{q}$ for any Schwartz function $f$ if and only if $\gamma-d / q \notin \mathbb{Z}_{+}$and $I=U_{\Omega, q /(q-1)}$.

(ii) If $q=\infty$ and $\gamma \notin \mathbb{Z}_{+}$, then the Fourier transform of If belongs to $L^{\infty}$ for any Schwartz function $f$ if and only if $I=U_{\Omega, 1}$.

(iii) If $q=\infty$ and $\gamma \in \mathbb{Z}_{+}$, then the Fourier transform of If belongs to $L^{\infty}$ for any Schwartz function $f$ if and only if

$$
\widehat{I f}(\xi)=\widehat{U_{\Omega, 1} f}(\xi)+\sum_{|\mathbf{i}|=-\gamma} \frac{\partial^{\mathbf{i}} \hat{f}(\mathbf{0})}{\mathbf{i} !} g_{\mathbf{i}}(\xi)
$$

for some bounded homogeneous functions $g_{\mathbf{i}},|\mathbf{i}|=-\gamma$, of degree 0 .

Proof (i) The sufficiency follows from Theorem 3.1 and Corollary 3.2. Now we prove the necessity. As every $q$-integrable function belong to $K_{1}$, similar to the argument used in the proof of Lemma 2.12, we can find functions $g_{\mathbf{i}} \in K_{1}$, $|\mathbf{i}| \leq$ $N$, such that

$$
\widehat{I f}(\xi)=\mathcal{F}\left(U_{\Omega, q /(q-1)} f\right)(\xi)+\sum_{|\mathbf{i}| \leq N} \frac{\partial^{\mathbf{i}} \hat{f}(\mathbf{0})}{\mathbf{i} !} g_{\mathbf{i}}(\xi) .
$$

Let $\psi_{\mathbf{j}}, j \in \mathbb{Z}_{+}^{d}$ be defined as in (2.29). Replacing $f$ by $\psi_{\mathbf{j}}$ with $|\mathbf{j}| \leq N$ and using (2.30) gives

$$
\begin{aligned}
& \widehat{I \psi_{\mathbf{j}}}(\xi)=\left(\widehat{\psi}_{\mathbf{j}}(\xi)-\sum_{|\mathbf{i}| \leq-\gamma-d / q} \frac{\partial^{\mathbf{i}} \hat{\psi}_{\mathbf{j}}(\mathbf{0})}{\mathbf{i} !} \xi^{\mathbf{i}}\right) \Omega(\xi)+g_{\mathbf{j}}(\xi) \\
& = \begin{cases}\frac{\xi \mathbf{j}}{\mathbf{j} !}(\phi(\xi)-1) \Omega(\xi)+g_{\mathbf{j}}(\xi) & \text { if }|\mathbf{j}| \leq \gamma-d / q, \\
\xi_{\mathbf{j} !}^{\mathbf{j}} \phi(\xi) \Omega(\xi)+g_{\mathbf{j}}(\xi) & \text { if }|\mathbf{j}|>\gamma-d / q .\end{cases}
\end{aligned}
$$

Note that $\frac{\xi^{\mathbf{j}}}{\mathbf{j} !}(\phi(\xi)-1) \Omega(\xi) \in L^{q}$ when $|\mathbf{j}|<\gamma-d / q$, and $\frac{\xi^{\mathbf{j}}}{\mathbf{j} !} \phi(\xi) \Omega(\xi) \in L^{p}$ when $|\mathbf{j}|>\gamma-d / q$. This, together with (3.34) and the assumption that $\widehat{I \psi_{\mathbf{j}}} \in$ $L^{q}$, proves that

$$
g_{\mathbf{j}} \in L^{q} \quad \text { for all } \mathbf{j} \in \mathbb{Z}_{+}^{d} \quad \text { with } \gamma-d / q \neq|\mathbf{j}| \leq N .
$$


By the homogeneous property of the linear map $I$, the functions $g_{\mathbf{i}},|\mathbf{i}| \leq N$, are homogeneous of degree $-\gamma+|\mathbf{i}|$, i.e.,

$$
g_{\mathbf{i}}(t \xi)=t^{-\gamma+|\mathbf{i}|} g_{\mathbf{i}}(\xi), \quad \text { for all } t>0 .
$$

Combining (3.35) and (3.36) proves that $g_{\mathbf{j}}=0$ for all $\mathbf{j} \in \mathbb{Z}_{+}^{d}$ with $\gamma-d / q \neq$ $|\mathbf{j}| \leq N$, and the desired conclusion $\widehat{I f}(\xi)=\mathcal{F}\left(U_{\Omega, q /(q-1)} f\right)(\xi)$ for all $f \in \mathcal{S}$ when $\gamma-d / q \notin \mathbb{Z}_{+}$.

Now it suffices to prove that $\gamma-d / q \notin \mathbb{Z}_{+}$. Suppose on the contrary that $\gamma-d / q \in \mathbb{Z}_{+}$. Then $1<q<\infty$ as $\gamma \notin \mathbb{Z}$. By (3.34) and the assumption on the linear map $I$, we have

$$
\int_{\xi \notin \operatorname{supp} \phi}\left|g_{\mathbf{j}}(\xi)-\xi^{\mathbf{j}} \Omega(\xi) / \mathbf{j} !\right|^{q} d \xi=\int_{\xi \notin \operatorname{supp} \phi}\left|\widehat{I \psi_{\mathbf{j}}}(\xi)\right|^{q} d \xi<\infty
$$

for all $\mathbf{j} \in \mathbb{Z}_{+}^{d}$ with $|\mathbf{j}|=\gamma-d / q$. This, together with (3.36) and the fact that the support $\operatorname{supp} \phi$ of the function $\phi$ is a bounded set, implies that $g_{\mathbf{j}}(\xi)-$ $\xi^{\mathbf{j}} \Omega(\xi) / \mathbf{j} !=0$ for all $\mathbf{j} \in \mathbb{Z}_{+}^{d}$ with $|\mathbf{j}|=\gamma-d / q$. By substituting the above equality for $g_{\mathbf{j}}$ into (3.34) we obtain

$$
\widehat{I \psi_{\mathbf{j}}}(\xi)=\phi(\xi) \xi^{\mathbf{j}} \Omega(\xi) / \mathbf{j} !
$$

for all $\mathbf{j} \in \mathbb{Z}_{+}^{d}$ with $|\mathbf{j}|=\gamma-d / q$. This leads to a contradiction, as $\widehat{\operatorname{I\psi }_{\mathbf{j}}}(\xi) \in L^{q}$ by the assumption on the linear map $I$, and $\phi(\xi) \xi^{\mathbf{j}} \Omega(\xi) / \mathbf{j} ! \notin L^{q}$ by direction computation.

(ii) and (iii) The necessity is true by (3.32) and Theorem 3.1, while the sufficiency follows from (3.33)-(3.36).

\subsection{Proof of Theorem 1.2}

The conclusions in Theorem 1.2 follow easily from (2.47), (3.2), Theorem 3.7 and Corollary 3.4.

\section{Sparse stochastic processes}

In this section, we will prove Theorem 1.3 and fully characterize the generalized random process $P_{\gamma} w$, which is a solution of the stochastic partial differential equation (1.3). In particular, we provide its characteristic functional and its pointwise evaluation.

\subsection{Proof of Theorem 1.3}

To prove Theorem 1.3, we recall the Levy continuity theorem, and a fundamental theorem about the characteristic functional of a generalized random process.

Lemma 4.1 [5] Let $\xi_{k}, k \geq 1$, be a sequence of random variables whose characteristic functions are denoted by $\mu_{k}(t)$. If $\lim _{k \rightarrow \infty} \mu_{k}(t)=\mu_{\infty}(t)$ for some 
continuous function $\mu_{\infty}(t)$ on the real line, then $\xi_{k}$ converges to a random variable $\xi_{\infty}$ in distribution whose characteristic function $\mathbf{E}\left(e^{-i t \xi_{\infty}}\right)$ is $\mu_{\infty}(t)$.

In the study of generalized random processes, the characteristic functional plays a similar role to the characteristic function of a random variable [6]. The idea is to formally specify a generalized random process $\Phi$ by its characteristic functional $\mathcal{Z}_{\Phi}$ given by

$$
\mathcal{Z}_{\Phi}(f):=\mathbf{E}\left(e^{-i \Phi(f)}\right)=\int_{\mathbb{R}} e^{-i x} d P(x), \quad f \in \mathcal{D},
$$

where $P(x)$ denotes the probability that $\Phi(f)<x$. For instance, we can show [14] that the characteristic functional $\mathcal{Z}_{w}$ of the white Poisson noise (1.13) is given by

$$
\mathcal{Z}_{w}(f)=\exp \left(\lambda \int_{\mathbb{R}^{d}} \int_{\mathbb{R}}\left(e^{-i a f(\mathbf{x})}-1\right) d P(a) d \mathbf{x}\right), \quad f \in \mathcal{D} .
$$

The characteristic functional $\mathcal{Z}_{\Phi}$ of a generalized random process $\Phi$ is a functional from $\mathcal{D}$ to $\mathbb{C}$ that is continuous and positive-definite, and satisfies $\mathcal{Z}_{\Phi}(0)=1$. Here the continuity of a functional $L$ from $\mathcal{D}$ to $\mathbb{C}$ means that $\lim _{k \rightarrow \infty} L\left(f_{k}\right)=L(f)$ if $f_{k} \in \mathcal{D}$ tends to $f \in \mathcal{D}$ in the topology of the space $\mathcal{D}$, while a functional $L$ from $\mathcal{D}$ to $\mathbb{C}$ is said to be positive-definite if

$$
\sum_{j, k=1}^{n} L\left(f_{j}-f_{k}\right) c_{j} \bar{c}_{k} \geq 0
$$

for any $f_{1}, \ldots, f_{n} \in \mathcal{D}$ and any complex numbers $c_{1}, \ldots, c_{n}$. The remarkable aspect of the theory of generalized random processes is that specification of $\mathcal{Z}_{\Phi}$ is sufficient to define a process in a consistent and unambiguous way. This is stated in the fundamental Minlos-Bochner theorem.

Theorem 4.2 [6] Let $L$ be a positive-definite continuous functional on $\mathcal{D}$ such that $L(0)=1$. Then there exists a generalized random process $\Phi$ whose characteristic functional is $L$. Moreover for any $f_{1}, \ldots, f_{n} \in \mathcal{D}$, we may take the positive measure $P\left(x_{1}, \ldots, x_{n}\right)$ as the distribution function of the random variable $\Phi\left(f_{1}\right), \ldots, \Phi\left(f_{n}\right)$, where the Fourier transform of the positive measure $P\left(x_{1}, \ldots, x_{n}\right)$ is $L\left(y_{1} f_{1}+\cdots+y_{n} f_{n}\right)$, i.e.,

$$
L\left(y_{1} f_{1}+\ldots+y_{n} f_{n}\right)=\int_{\mathbb{R}^{n}} \exp \left(-i\left(x_{1} y_{1}+\ldots+x_{n} y_{n}\right)\right) d P\left(x_{1}, \ldots, x_{n}\right) .
$$

We are now ready to prove Theorem 1.3.

Proof of Theorem 1.3 Let $N \geq 1$ and $\varphi$ be a $C^{\infty}$ function supported in $B(\mathbf{0}, 2)$ and taking the value one in $B(\mathbf{0}, 1)$. For any $f \in \mathcal{D}$, define a sequence of random variables $\Phi_{\gamma, N}(f)$ associated with $f$ by

$$
\Phi_{\gamma, N}(f):=\sum_{k} a_{k} \varphi\left(\mathbf{x}_{k} / N\right) I_{\gamma, 1} f\left(\mathbf{x}_{k}\right),
$$


where the $a_{k}$ 's are i.i.d. random variables with probability distribution $P(a)$, and where the $\mathbf{x}_{k}$ 's are random point locations in $\mathbb{R}^{n}$ which are mutually independent and follow a spatial Poisson distribution with Poisson parameter $\lambda>0$. We will show that $\Phi_{\gamma, N}, N \geq 1$, define a sequence of generalized random processes, whose limit $P_{\gamma} w(f):=\sum_{k} a_{k} I_{\gamma, 1}(f)\left(\mathbf{x}_{k}\right)$ is a solution of the stochastic partial differential equation (1.3).

As $\varphi$ is a continuous function supported on $B(\mathbf{0}, 2)$,

$$
\Phi_{\gamma, N}(f)=\sum_{\mathbf{x}_{k} \in B(\mathbf{0}, 2 N)} a_{k} \varphi\left(\mathbf{x}_{k} / N\right) I_{\gamma, 1} f\left(\mathbf{x}_{k}\right) .
$$

Recall that $I_{\gamma, 1} f$ is continuous on $\mathbb{R}^{d} \backslash\{\mathbf{0}\}$ by Corollary 3.6. Then the summation of the right-hand side of (4.5) is well-defined whenever there are finitely many $\mathbf{x}_{k}$ in $B(\mathbf{0}, 2 N)$ with none of them belonging to $B(\mathbf{0}, \epsilon), \epsilon>0$. Note that the probability that at least one of $\mathbf{x}_{k}$ lies in the small neighbor $B(\mathbf{0}, \epsilon)$ is equal to

$$
\sum_{n=1}^{\infty} e^{-\lambda|B(\mathbf{0}, \epsilon)|} \frac{(\lambda|B(\mathbf{0}, \epsilon)|)^{n}}{n !}=1-e^{-\lambda|B(\mathbf{0}, \epsilon)|} \rightarrow 0 \quad \text { as } \epsilon \rightarrow 0 .
$$

We then conclude that $\Phi_{\gamma, N}(f)$ is well-defined and $\Phi_{\gamma, N}(f)<\infty$ with probability one.

Denote the characteristic function of the random variable $\Phi_{\gamma, N}(f)$ by $E_{\gamma, N, f}(t)$ :

$$
E_{\gamma, N, f}(t)=\mathbf{E}\left(e^{-i t \Phi_{\gamma, N}(f)}\right)=\mathbf{E}\left(e^{-i \Phi_{\gamma, N}(t f)}\right) .
$$

Applying the same technique as in [12, Appendix B], we can show that

$$
E_{\gamma, N, f}(t)=\exp \left(\int_{\mathbb{R}^{d}} \int_{\mathbb{R}}\left(e^{-i a t \varphi(\mathbf{x} / N) I_{\gamma, 1} f(\mathbf{x})}-1\right) d P(a) d \mathbf{x}\right) .
$$

Moreover, the functional $E_{\gamma, N, f}(t)$ is continuous about $t$ by the dominated convergence theorem, because

$$
\left|e^{-i a t \varphi(\mathbf{x} / N) I_{\gamma, 1} f(\mathbf{x})}-1\right| \leq|a||t|\left|I_{\gamma, 1} f(\mathbf{x})\right|
$$

and

$$
\int_{\mathbb{R}^{d}} \int_{\mathbb{R}}|a|\left|I_{\gamma, 1} f(\mathbf{x})\right| d P(a) d \mathbf{x}=\left(\int_{\mathbb{R}}|a| d P(a)\right) \times\left(\int_{\mathbb{R}^{d}}\left|I_{\gamma, 1} f(\mathbf{x})\right| d \mathbf{x}\right)<\infty
$$

by Corollary 3.6 and the assumption on the distribution $P$.

Clearly the random variable $\Phi_{\gamma, N}(f)$ is linear about $f \in \mathcal{D}$; i.e.,

$$
\Phi_{\gamma, N}(\alpha f+\beta g)=\alpha \Phi_{\gamma, N}(f)+\beta \Phi_{\gamma, N}(g) \quad \text { for all } f, g \in \mathcal{D} \text { and } \alpha, \beta \in \mathbb{R}
$$


For any sequence of functions $f_{k}$ in $\mathcal{D}$ that converges to $f_{\infty}$ in the topology of $\mathcal{D}$, it follows from Theorem 3.5 and Corollary 3.6 that $\lim _{k \rightarrow \infty} \| I_{\gamma, 1} f_{k}-$ $I_{\gamma, 1} f_{\infty} \|_{1}=0$. Therefore

$$
\begin{aligned}
& \mid \int_{\mathbb{R}^{d}} \int_{\mathbb{R}}\left(e^{-i a t \varphi(\mathbf{x} / N) I_{\gamma, 1} f_{k}(\mathbf{x})}-1\right) d P(a) d \mathbf{x} \\
& \quad-\int_{\mathbb{R}^{d}} \int_{\mathbb{R}}\left(e^{-i a t \varphi(\mathbf{x} / N) I_{\gamma, 1} f_{\infty}(\mathbf{x})}-1\right) d P(a) d \mathbf{x} \mid \\
& \quad \leq|t|\left(\int_{\mathbb{R}}|a| d P(a)\right)\left(\int_{\mathbb{R}^{d}} \varphi(\mathbf{x} / N)\left|I_{\gamma, 1} f_{k}(\mathbf{x})-I_{\gamma, 1} f_{\infty}(\mathbf{x})\right| d \mathbf{x}\right) \\
& \quad \rightarrow 0 \quad \text { as } k \rightarrow \infty,
\end{aligned}
$$

which implies that the characteristic function of $\Phi_{\gamma, N}\left(f_{k}\right)$ converges to the continuous characteristic function of $\Phi_{\gamma, N}\left(f_{\infty}\right)$. Hence the random variable $\Phi_{\gamma, N}\left(f_{k}\right)$ converges to $\Phi_{\gamma, N}\left(f_{\infty}\right)$ by Lemma 4.1 , which in turn implies that $\Phi_{\gamma, N}$ is continuous on $\mathcal{D}$.

Set

$$
L_{\gamma, N}(f)=E_{\gamma, N, f}(1)
$$

For any sequence $c_{l}, 1 \leq l \leq n$, of complex numbers and $f_{l}, 1 \leq l \leq n$, of functions in $\mathcal{D}$,

$$
\begin{aligned}
\sum_{1 \leq l, l^{\prime} \leq n} L_{\gamma, N}\left(f_{l}-f_{l^{\prime}}\right) c_{l} \overline{c_{l^{\prime}}} & =\mathbf{E}\left(\sum_{l, l^{\prime}=1}^{n} e^{-i \Phi_{\gamma, N}\left(f_{l}-f_{l^{\prime}}\right)} c_{l} \overline{c_{l^{\prime}}}\right) \\
& =\mathbf{E}\left(\left|\sum_{l=1}^{n} c_{l} e^{-i \Phi_{\gamma, N}\left(f_{l}\right)}\right|^{2}\right) \geq 0
\end{aligned}
$$

which implies that $L_{\gamma, N}$ is positive-definite. By Theorem 4.2, we conclude that $\Phi_{\gamma, N}$ defines a generalized random process with characteristic functional $L_{\gamma, N}$.

Now we consider the limit of the above family of generalized random processes $\Phi_{\gamma, N}, N \geq 1$. By Corollary 3.6, $I_{\gamma, 1} f$ is integrable for all $f \in \mathcal{D}$. Then

$$
\lim _{N \rightarrow+\infty} E_{\gamma, N, f}(t)=\exp \left(\int_{\mathbb{R}^{d}} \int_{\mathbb{R}}\left(e^{-i a t I_{\gamma, 1} f(\mathbf{x})}-1\right) d P(a) d \mathbf{x}\right)=: E_{\gamma, f}(t)
$$

Clearly $E_{\gamma, f}(0)=1$ and $E_{\gamma, f}(t)$ is continuous as $I_{\gamma, 1}(f)$ is integrable. Therefore by Lemma 4.1, $\Phi_{\gamma, N}(f)$ converges to a random variable, which is denoted by $P_{\gamma}(f):=\sum_{k} a_{k} I_{\gamma, 1} f\left(\mathbf{x}_{k}\right)$, in distribution. 
As $I_{\gamma, 1} f$ is a continuous map from $\mathcal{D}$ to $L^{1}$, then $\lim _{k \rightarrow \infty} \| I_{\gamma, 1} f_{k}-$ $I_{\gamma, 1} f_{\infty} \|_{1}=0$ whenever $f_{k}$ converges to $f$ in $\mathcal{D}$. Hence

$$
\begin{aligned}
& \mid \int_{\mathbb{R}^{d}} \int_{\mathbb{R}}\left(e^{-i a t I_{\gamma, 1} f_{k}(\mathbf{x})}-1\right) d P(a) d \mathbf{x} \\
& \quad-\int_{\mathbb{R}^{d}} \int_{\mathbb{R}}\left(e^{-i a t I_{\gamma, 1} f_{\infty}(\mathbf{x})}-1\right) d P(a) d \mathbf{x} \mid \\
& \quad \leq|t|\left(\int_{\mathbb{R}}|a| d P(a)\right)\left(\int_{\mathbb{R}^{d}}\left|I_{\gamma, 1} f_{k}(\mathbf{x})-I_{\gamma, 1} f_{\infty}(\mathbf{x})\right| d \mathbf{x}\right) \\
& \quad \rightarrow 0 \quad \text { as } k \rightarrow \infty,
\end{aligned}
$$

which implies that the characteristic function of $P_{\gamma}\left(f_{k}\right)$ converges to the characteristic function of $P_{\gamma}\left(f_{\infty}\right)$ (which is also continuous), and hence $P_{\gamma}\left(f_{k}\right)$ converges to $P_{\gamma}\left(f_{\infty}\right)$ in distribution by Lemma 4.1. From the above argument, we see that $P_{\gamma}(f)$ is continuous about $f \in \mathcal{D}$.

Define $L_{\gamma}(f)=E_{\gamma, f}(1)$. From (4.10) and (4.11), we see that

$$
\sum_{1 \leq l, l^{\prime} \leq n} L_{\gamma}\left(f_{l}-f_{l^{\prime}}\right) c_{i} \overline{c_{i^{\prime}}}=\lim _{N \rightarrow \infty} \sum_{1 \leq l, l^{\prime} \leq n} L_{\gamma, N}\left(f_{l}-f_{l^{\prime}}\right) c_{i} \overline{c_{l^{\prime}}} \geq 0
$$

for any sequence $c_{l}, 1 \leq l \leq n$, of complex numbers and $f_{l}, 1 \leq l \leq n$, of functions in $\mathcal{D}$. Therefore by Theorem 4.2, $P_{\gamma} w$ defines a generalized random process with its characteristic functional given by

$$
\mathcal{Z}_{P_{\gamma} w}(f)=\exp \left(\int_{\mathbb{R}^{d}} \int_{\mathbb{R}}\left(e^{-i a I_{\gamma, 1} f(\mathbf{x})}-1\right) d P(a) d \mathbf{x}\right) .
$$

\subsection{Pointwise evaluation}

In this section, we consider the pointwise characterization of the generalized random process $P_{\gamma} w$.

Theorem 4.3 Let $\gamma, \lambda, P(a), P_{\gamma} w$ be as in Theorem 1.3, and $I_{\gamma, 1}$ be defined as in (1.12). Then

$$
P_{\gamma} w\left(\mathbf{y}_{0}\right):=\lim _{N \rightarrow \infty} P_{\gamma} w\left(g_{N, \mathbf{y}_{0}}\right)
$$

is a random variable for every $\mathbf{y}_{0} \in \mathbb{R}^{d}$ whose characteristic function is given by

$$
\mathbf{E}\left(e^{-i t P_{\gamma} w\left(\mathbf{y}_{0}\right)}\right)=\exp \left(\lambda \int_{\mathbb{R}} \int_{\mathbb{R}}\left(e^{-i a t H_{\mathbf{y}_{0}}(\mathbf{x})}-1\right) d \mathbf{x} d P(a)\right), t \in \mathbb{R},
$$

where $g \in \mathcal{D}$ satisfies $\int_{\mathbb{R}^{d}} g(\mathbf{x}) d \mathbf{x}=1, g_{N, \mathbf{y}_{0}}(\mathbf{x})=N^{d} g\left(N\left(\mathbf{x}-\mathbf{y}_{0}\right)\right)$, and

$$
\widehat{H_{\mathbf{y}_{0}}}(\xi)=\left(e^{i\left(\mathbf{y}_{0}, \xi\right\rangle}-\sum_{|\mathbf{i}| \leq \gamma} \frac{\left(i \mathbf{y}_{0}\right)^{\mathbf{i} \xi} \xi}{\mathbf{i} !}\right)\|\xi\|^{-\gamma} .
$$


An interpretation is that the random variable $P_{\gamma} w\left(\mathbf{y}_{0}\right)$ in (4.15) and its characteristic function $\mathbf{E}\left(e^{-i t P_{\gamma} w\left(\mathbf{y}_{0}\right)}\right)$ in (4.16) correspond formally to setting $f=\delta\left(\cdot-\mathbf{y}_{0}\right)$ (the delta distribution) in (1.18) and (1.19), respectively.

To prove Theorem 4.3, we need a technical lemma.

Lemma 4.4 Let $\gamma$ be a positive non-integer number, $g \in \mathcal{D}$ satisfy $\int_{\mathbb{R}^{d}} g(\mathbf{x}) d \mathbf{x}=$ 1 , and $H_{\mathbf{y}_{0}}$ be defined in (4.17). Then

$$
\lim _{N \rightarrow \infty}\left\|I_{\gamma, 1} g_{N, \mathbf{y}_{0}}-H_{\mathbf{y}_{0}}\right\|_{1}=0
$$

for all $\mathbf{y}_{0} \in \mathbb{R}^{d}$, where $g_{N, \mathbf{y}_{0}}(\mathbf{x})=N^{d} g\left(N\left(\mathbf{x}-\mathbf{y}_{0}\right)\right)$.

Proof Let $K_{\mathbf{j}}$ be the inverse Fourier transform of $(i \xi)^{\mathbf{j}}\|\xi\|^{-\gamma}$ and $k_{1}$ be the integral part of the positive non-integer number $\gamma$. Then from the argument in the proof of Theorem 3.5,

$$
H_{\mathbf{y}}(\mathbf{x})= \begin{cases}\sum_{|\mathbf{j}|=k_{1}} \frac{k_{1}}{\mathbf{j} !} \int_{0}^{1}\left(K_{\mathbf{j}}(\mathbf{x}-t \mathbf{y})-K_{\mathbf{j}}(\mathbf{x})\right)(-\mathbf{y})^{\mathbf{j}}(1-t)^{k_{1}-1} d t & \text { if } k_{1} \geq 1 \\ K_{\mathbf{0}}(\mathbf{x}-\mathbf{y})-K_{\mathbf{0}}(\mathbf{x}) & \text { if } k_{1}=0\end{cases}
$$

Therefore for $\mathbf{y}_{0} \neq 0$,

$$
\begin{aligned}
& \left\|I_{\gamma, 1} g_{N, \mathbf{y}_{0}}-H_{\mathbf{y}_{0}}\right\|_{1} \\
& \leq C \sum_{|\mathbf{j}|=k_{1}} \int_{\mathbb{R}^{d}} \int_{0}^{1} \int_{\mathbb{R}^{d}} \mid\left(K_{\mathbf{j}}(\mathbf{x}-t \mathbf{y})-K_{\mathbf{j}}(\mathbf{x})\right) \mathbf{y}^{\mathbf{j}} \\
& -\left(K_{\mathbf{j}}\left(\mathbf{x}-t \mathbf{y}_{0}\right)-K_{\mathbf{j}}(\mathbf{x})\right) \mathbf{y}_{0}^{\mathbf{j}}|| g_{N, \mathbf{y}_{0}}(\mathbf{y}) \mid d \mathbf{y} d t d \mathbf{x} \\
& \leq C \sum_{|\mathbf{j}|=k_{1}} \int_{\mathbb{R}^{d}} \int_{0}^{1} \int_{\mathbb{R}^{d}}\left|K_{\mathbf{j}}(\mathbf{x}-t \mathbf{y})-K_{\mathbf{j}}\left(\mathbf{x}-t \mathbf{y}_{0}\right)\right|\|\mathbf{y}\|^{k_{1}}\left|g_{N, \mathbf{y}_{0}}(\mathbf{y})\right| d \mathbf{y} d t d \mathbf{x} \\
& +C \sum_{|\mathbf{j}|=k_{1}} \int_{\mathbb{R}^{d}} \int_{0}^{1} \int_{\mathbb{R}^{d}}\left|K_{\mathbf{j}}\left(\mathbf{x}-t \mathbf{y}_{0}\right)-K_{\mathbf{j}}(\mathbf{x})\left\|\mathbf{y}^{\mathbf{j}}-\mathbf{y}_{0}^{\mathbf{j}}\right\| g_{N, \mathbf{y}_{0}}(\mathbf{y})\right| d \mathbf{y} d t d \mathbf{x} \\
& \leq C \int_{0}^{1} \int_{\mathbb{R}^{d}}\left(t\left\|\mathbf{y}-\mathbf{y}_{0}\right\|\right)^{\gamma-k_{1}}\left(\left\|\mathbf{y}_{0}\right\|^{k_{1}}+\left\|\mathbf{y}-\mathbf{y}_{0}\right\|^{k_{1}}\right)\left|g_{N, \mathbf{y}_{0}}(\mathbf{y})\right| d \mathbf{y} d t \\
& +C \int_{0}^{1} \int_{\mathbb{R}^{d}}\left(t\left\|\mathbf{y}_{0}\right\|\right)^{\gamma-k_{1}}\left(\left\|\mathbf{y}_{0}\right\|^{k_{1}-1}\left\|\mathbf{y}-\mathbf{y}_{0}\right\|+\left\|\mathbf{y}-\mathbf{y}_{0}\right\|^{k_{1}}\right)\left|g_{N, \mathbf{y}_{0}}(\mathbf{y})\right| d \mathbf{y} d t \\
& \rightarrow 0 \quad \text { as } N \rightarrow \infty
\end{aligned}
$$


if $k_{1} \geq 1$, and

$$
\begin{aligned}
& \left\|I_{\gamma, 1} g_{N, \mathbf{y}_{0}}-H_{\mathbf{y}_{0}}\right\|_{1} \\
& \leq \int_{\mathbb{R}^{d}} \int_{\mathbb{R}^{d}} \mid K_{\mathbf{0}}(\mathbf{x}-\mathbf{y})-K_{\mathbf{0}}\left(\mathbf{x}-\mathbf{y}_{0}\right) \| g_{N, \mathbf{y}_{0}}(\mathbf{y}) d \mathbf{y} d \mathbf{x} \\
& \leq \int_{\mathbb{R}^{d}}\left(\int_{\|\mathbf{x}-\mathbf{y}\| \geq 2\left\|\mathbf{y}-\mathbf{y}_{0}\right\|}\left|K_{\mathbf{0}}(\mathbf{x}-\mathbf{y})-K_{\mathbf{0}}\left(\mathbf{x}-\mathbf{y}_{0}\right)\right| d \mathbf{x}\right. \\
& \left.\quad+\int_{\|\mathbf{x}-\mathbf{y}\| \leq 2\left\|\mathbf{y}-\mathbf{y}_{0}\right\|}\left|K_{\mathbf{0}}(\mathbf{x}-\mathbf{y})\right|+\left|K_{\mathbf{0}}\left(\mathbf{x}-\mathbf{y}_{0}\right)\right| d \mathbf{x}\right)\left|g_{N, \mathbf{y}_{0}}(\mathbf{y})\right| d \mathbf{y} \\
& \leq C N^{d} \int_{\mathbb{R}^{d}}\left\|\mathbf{y}-\mathbf{y}_{0}\right\|^{\gamma}\left|g\left(N\left(\mathbf{y}-\mathbf{y}_{0}\right)\right)\right| d \mathbf{y} \\
& =C N^{-\gamma} \int_{\mathbb{R}^{d}}\|\mathbf{z}\|^{\gamma}|g(\mathbf{z})| d \mathbf{z} \rightarrow 0 \quad \text { as } N \rightarrow 0,
\end{aligned}
$$

if $k_{1}=0$. This shows that (4.18) for $\mathbf{y}_{0} \neq \mathbf{0}$.

The limit in (4.18) for $\mathbf{y}_{0}=\mathbf{0}$ can be proved by using a similar argument, the detail of which are omitted here.

Proof of Theorem 4.3 By Lemma 4.4 and the dominated convergence theorem,

$$
\lim _{N \rightarrow \infty} \int_{\mathbb{R}^{d}} \int_{\mathbb{R}}\left(e^{-i a t I_{\gamma, 1} g_{N, \mathbf{y}_{0}}(\mathbf{x})}-1\right) d P(a) d \mathbf{x}=\int_{\mathbb{R}^{d}} \int_{\mathbb{R}}\left(e^{-i a t H_{\mathbf{y}_{0}}(\mathbf{x})}-1\right) d P(a) d \mathbf{x}
$$

for all $t \in \mathbb{R}$. Moreover as $H_{\mathbf{y}_{0}}$ is integrable from Corollary 3.6 and Lemma 4.4, the function $\int_{\mathbb{R}^{d}} \int_{\mathbb{R}}\left(e^{-i a t I_{\gamma, 1} H_{\mathbf{y}_{0}}(\mathbf{x})}-1\right) d P(a) d \mathbf{x}$ is continuous about $t$. Therefore (4.15) and (4.16) follows from Lemma 4.1.

Acknowledgements This work was done when the first named author was visiting Ecole Polytechnique Federale de Lausanne on his sabbatical leave. He would like to thank Professors Michael Unser and Martin Vetterli for the hospitality and fruitful discussions.

Open Access This article is distributed under the terms of the Creative Commons Attribution Noncommercial License which permits any noncommercial use, distribution, and reproduction in any medium, provided the original author(s) and source are credited.

\section{References}

1. Blu, T., Unser, M.: Self-similarity Part II: optimal estimation of fractal processes. IEEE Trans. Signal Process. 55, 1364-1378 (2007)

2. Caffarelli, L.A., Salsa, S., Silvestre, L.: Regularity estimates for the solution and the free boundary to the obstacle problem for the fractional Laplacian. Invent. Math. 171, 425-461 (2008)

3. Chen, Z.-Q., Kim, P., Song, R.: Heat kernel estimates for Dirichlet fractional Laplacian. J. Eur. Math. Soc. 12, 1307-1329 (2010) 
4. Duchon, J.: Splines minimizing rotation-invariant semi-norms in Sobolev spaces. In: eds. Schempp, W., Zeller, K.: Constructive Theory of Functions of Several Variables, pp. 85-100. Springer (1977)

5. Fristedt, B., Gray, L.: A Modern Approach to Probability Theory. Birkhauser (1997)

6. Gelfand, I.M., Vilenkin, N.Y.: Generalized Functions-Vol 4: Applications of Harmonic Analysis. Academic Press (1964)

7. Hörmander, L.: The Analysis of Linear Partial Differential Operators I. Springer (1989)

8. Mandelbrot, B.B., Van Ness, J.W.: Fractional Brownian motions, fractional noises and applications. SIAM Rev. 10, 422-437 (1968)

9. Riesz, M.: L'intégrale de Riemann-Liouville et le probléme de Cauchy. Acta Math. 81, 1-223 (1949)

10. Silvestre, L.: Hölder estimates for solutions of integro-differential equations like the fractional Laplace. Indiana Univ. Math. J. 55, 1155-1174 (2006)

11. Stein, E.: Singular Integrals and Differentiability Properties of Functions. Princeton University Press, Princeton, NJ (1970)

12. Tafti, P.D., Van De Ville, D., Unser, M.: Invariances, Laplacian-like wavelet bases, and the whitening of fractal processes. IEEE Trans. Image Process. 18, 689-702 (2009)

13. Tafti, P.D., Unser, M.: Fractional Brownian vector fields. SIAM J. Multiscale Model. Simul. 8, 1645-1670 (2010)

14. Unser, M., Tafti, P.D.: Stochastic models for sparse and piecewise-smooth signals. IEEE Trans. Signal Process. 59, 989-1006 (2011) 